http://dx.doi.org/10.18778/0208-6107.32.13

\author{
Pedro Schmechtig \\ Institut für Philosophie \\ Technische Universität Dresden \\ Pedro.schmechtig@gmx.de
}

\title{
KOGNITIVER RATSCHLAG, TESTIMONIALE AUTORITÄTEN UND DER WERT EPISTEMISCHER WEISHEIT
}

\begin{abstract}
Zusammenfassung
Der vorliegende Aufsatz beschäftigt sich mit der Frage, welche Rolle Weisheit in der Erkenntnistheorie spielt. Zu Beginn skizziere ich kurz den allgemeinen Rahmen der Untersuchung. Damit verbunden ist ein konkreter Vorschlag zur begrifflichen Bestimmung epistemischer Weisheit. Dieser Vorschlag wird im Hinblick auf drei unterschiedliche Aspekte der Zuschreibung epistemischer Weisheit (ontologisch, epistemisch, axiologisch) näher erläutert. Im Anschluss daran wird die zentrale These genauer diskutiert, die hinter der vorgeschlagenen Begriffsbestimmung steht. Demnach ist epistemische Weisheit eine testimoniale Aktivität, die eine interpersonale Struktur aufweist. Gemäß dieser Sichtweise sind weise (kognitive) Ratgeber als testimoniale Autoritäten $\mathrm{zu}$ betrachten, deren kognitive Überlegenheit im Rahmen eines „AutoritätenRessourcen-Modells" der Erklärung personaler Weisheit zu analysieren ist. Ausgehend von diesem Modell lässt sich abschließend zeigen, dass Weisheit in Verbindung mit kognitiven Ratschlägen einen unverwechselbaren epistemischen Wert besitzt.
\end{abstract}

\section{Schlüsselwörter:}

kognitive Ratschlag, epistemische Autorität, epistemische Werte, epistemische Weisheit, MetaErkenntnistheorie, personale Weisheit, Wert der Weisheit

Es gibt ein anhaltendes Interesse in der Erkenntnistheorie, genauer zu untersuchen, welche kognitiven Zustände oder Aktivitäten einen epistemischen Wert besitzen. Neben den vielerorts bekannten Kandidaten wird manchmal auch Weisheit ins Auge gefasst. ${ }^{1}$ Dieser Vorschlag ist jedoch mit einer besonderen

\footnotetext{
${ }^{1}$ Vgl. hierzu: Jason Baehr, „Two Types of Wisdom,” Acta Analytica 27 (2012); Jason Beahr, „Sophia: Theoretical Wisdom and Contemporary Epistemology," in Virtues and Their Vices, hg. Kevin Timpe und Craig A. Boyd (New York: Oxford University Press, 2014); Stephen Grimm, „Wisdom,” Australasian Journal of Philosophy 93 (2015); Christos Kyriacou, „Ought to Believe,
} 
Schwierigkeit verbunden. Betrachtet man die alltagssprachliche Verwendung des Prädikats „weise sein“ fällt es ungleich schwerer, anzunehmen, es gebe so etwas wie eine propositionale „first-order“ Einstellung der Weisheit, die analog $\mathrm{zu}$ wahren Meinungen, Wissen oder Verstehen in direkter Form einer Person zugeschrieben werden kann.

Andererseits sind wir mit dem Phänomen des weisen (kognitiven) Ratgebers vertraut, d. h. wir kennen Situationen, in denen uns eine kognitiv überlegene Person einen Ratschlag erteilt, was in einer Situation epistemisch gesehen das Beste ist. Dieser Tatbestand legt die Vermutung nahe, dass der Wert der Weisheit auch im erkenntnistheoretischen Zusammenhang einen festen Platz hat.

Der vorliegende Aufsatz verfolgt das Ziel, zu klären, wie Weisheit im Rahmen des kognitiven Ratschlags ins Spiel kommt. Das weiterführende Interesse an dieser Untersuchung besteht in der Beantwortung der folgenden Frage: Nehmen wir an, es gelingt uns, Weisheit in dieser Form erkenntnistheoretisch zu lokalisieren. Welche Schlussfolgerungen lassen sich daraus für die Analyse des Werts epistemischer Weisheit ziehen? Oder anders gefragt: Welchen Wert hat Weisheit im Hinblick auf die Tatsache, dass weise Personen in der Lage sind, anderen Akteuren exzellente kognitive Ratschläge zu erteilen?

Der Plan zu dieser Arbeit sieht folgendermaßen aus: Im ersten Schritt (Abschnitt 1) skizziere ich den allgemeinen Rahmen der vorliegenden Untersuchung. Dieser Abschnitt schließt mit einem konkreten Vorschlag zur begrifflichen Bestimmung epistemischer Weisheit. Danach (Abschnitt 2) diskutiere ich unter drei unterschiedlichen Gesichtspunkten (ontologisch, epistemisch, axiologisch) einige Besonderheiten der Zuschreibung epistemischer Weisheit, so wie sie sich aus der vorgeschlagenen Begriffsbestimmung ergeben.

Anschließend versuche ich der für meine Zwecke leitenden These mehr Gewicht zu verleihen, wonach epistemische Weisheit eine testimoniale Aktivität ist, die dadurch, dass sie anderen Personen als Quelle ihrer Erkenntnisbemühungen dienen kann, eine interpersonale Struktur aufweist (Abschnitt 3). Dazu werde ich einige Überlegungen zum testimonialen Transfer von Benjamin McMyler (2011) aufgreifen. Ziel dieses Abschnittes ist es, in allgemeiner Form verständ-

Evidential Understanding and the Pursuit of Wisdom," in Epistemic Reasons, Epistemic Norms, and Epistemic Goals, hg. Martin Grajner und Pedro Schmechtig (Berlin und Boston: DeGruyter, 2016); Nenad Miscevic, „Wisdom, Understanding and Knowledge: A Virtue-Theoretic Proposal,” Acta Analytica 27 (2012); Wayne Riggs, „Understanding 'Virtue' and the Virtue of Understanding," in Intellectual Virtue: Perspectives from Ethics and Epistemology, Hg. Michael DePaul und Linda Zagzebski (New York: Oxford University Press, 2003); Robert C. Roberts und W. Jay Wood, Intellectual Virtues: An Essay in Regulative Epistemology (New York: Oxford University Press, 2007); Pedro Schmechtig, „Ist Weisheit ein epistemisches Ziel?”, Zeitschrift für Philosophische Forschung 69, Nr. 4 (2015); Linda Zagzebski, Virtues of the Mind (Cambridge, UK: Cambridge University Press, 1996); Denis Whitcomb, „Wisdom,” in Routledge Companion to Epistemology, hg. Sven Bernecker und Duncan Pritchard (London: Routledge, 2010). 
lich zu machen, inwiefern ein „Buck-Passing“-Ansatz der Erklärung testimonialer Rechtfertigung die besten Aussichten hat, den speziellen „secondhand" Charakter der testimonialen Quelle des weisen (kognitiven) Ratschlags erklären zu können. Darüber hinaus argumentiere ich in diesem Zusammenhang für die These, dass weise (kognitive) Ratgeber testimoniale Autoritäten sind, deren kognitive Überlegenheit im Rahmen eines „Autoritäten-Ressourcen-Modells“ der Erklärung personaler Weisheit zu analysieren ist (Abschnitt 4). Im abschließenden Teil bilanziere ich dann den Ertrag der vorangegangenen Diskussion für die Beantwortung der Frage, inwiefern Weisheit im Zusammenhang mit kognitiven Ratschlägen einen unverwechselbaren (epistemischen) Wert besitzt (Abschnitt 5).

\section{WIE LÄSST SICH WEISHEIT IN EPISTEMISCHER HINSICHT BESTIMMEN?}

Es liegt nahe, den Begriff der epistemischen Weisheit in Anklang an Aristoteles als eine Tugend oder Charaktereigenschaft zu begreifen, deren Besitz durch eine bestimmte Form von Wissen definiert ist. Legt man die aristotelische Unterteilung von dianoetischen und ethischen Tugenden zugrunde, ergeben sich zwei unterschiedliche Varianten der Analyse:

\section{(A) Weisheit als umfangreiches theoretisches Wissen \\ Eine Person S ist weise, gdw. S umfangreiches faktisches Wissen / Prinzipien- Wissen im Hinblick auf einen bestimmten Bereich hat (Wissenschaft, Politik, Literatur usw.).}

(B) Weisheit als (erfolgreiches) Wissen-wie man gut lebt

Eine Person S ist weise, gdw. S weiß wie man gut oder glücklich lebt und erfolgreich danach handelt.

Die gegenläufigen Intuitionen beider Modelle haben zu einer bis heute anhaltenden Auseinandersetzung geführt, was Weisheit als personale Eigenschaft auszeichnet. Nach aktuellem Stand erweist sich keiner der beiden Vorschläge als vollständig oder praktikabel. ${ }^{2}$

Der unbefriedigende Zustand dieser Diskussion ${ }^{3}$ gibt uns die Gelegenheit, über einen alternativen Zugang zur allgemeinen Bestimmung von Weisheit

2 Das Hauptproblem von (A) ist: Es gibt viele Personen, die über ein hohes Maß an faktischem Wissen verfügen, aber alles in allem nicht weise sind. Demgegenüber lautet der zentrale Einwand gegenüber (B): Eine weise Person sollte nicht nur wissen wie man gut lebt, sie muss auch wissen und/oder verstehen können, warum das so ist, d. h. sie sollte in theoretisch-reflexiver Weise erklären können, was genau ein gutes Leben kennzeichnet bzw. welche Gründe es gibt, in dieser oder jener Form leben zu wollen. Ein ausführlicherer Überblick über diese Diskussion findet sich in Schmechtig, „Ist Weisheit ein epistemisches Ziel?”.

${ }^{3}$ Eine solche agnostische Einschätzung der bestehenden Diskussion hat Sharon Ryan dazu geführt, eine „Hybrid Theory of Wisdom“ vorzuschlagen, Sharon Ryan, „What is Wisdom?”, Philosophical Studies 93, Nr. 2 (1999). Später hat Ryan jedoch selbst einräumen müssen, dass auch 
nachzudenken. Das Prädikat „weise sein“ lässt sich nicht nur auf Charaktereigenschaften, sondern auch auf Handlungen und insbesondere auf Entscheidungen anwenden. Infolgedessen bestünde die Möglichkeit, in einem ersten Schritt den wahrscheinlich einfacher zu handhabenden Begriff der weisen Entscheidung $\mathrm{zu}$ definieren, um anschließend auf dieser Grundlage zu einer gesicherten - nämlich an Entscheidungen orientierten - Verwendung des Prädikats „weise sein" zu gelangen, mit dessen Hilfe sich der Begriff der personalen Weisheit weniger umstritten analysieren lässt. ${ }^{4}$

Ich halte diese Strategie der Erklärung von Weisheit nicht nur für sinnvoll, sondern im Vergleich mit anderen Herangehensweisen für erfolgsversprechender. An dieser Stelle geht es mir jedoch nicht darum, weise Entscheidungen zu analysieren. Vielmehr möchte ich klären, was es im Rahmen einer solchen Untersuchungsstrategie bedeuten kann, dass wir Weisheit in epistemischer Hinsicht wertschätzen. Das grundlegende Problem in diesem Zusammenhang ist Folgendes: Gemäß dieser Strategie gibt es eine basale entscheidungstheoretische Dimension von Weisheit. Es ist jedoch fraglich, ob sich ein solcher Zugang zur Erklärung von Weisheit (wie auch immer er aussehen mag) auf epistemische Zusammenhänge übertragen lässt. Nach traditionellem Verständnis sind kognitive Zustände doxastischer Natur, d. h. sie sind nur dann in epistemischer Hinsicht signifikant - und besitzen folglich einen epistemischen Wert -, wenn sie direkt oder in abgewandelter Form dem Ziel der wahren Meinung dienen. Die gängige Sichtweise besagt: Aufgrund dieser Wahrheitszielorientierung beruhen epistemisch signifikante Überzeugungen nicht - zumindest nicht in aktiver Form - auf so etwas wie bewussten Entscheidungen.

Im Gegensatz dazu wird im Rahmen der vorgeschlagenen Strategie behauptet, dass epistemische Weisheit darauf zurückzuführen ist, dass ein exzellenter kognitiver Ratgeber in der Lage ist, regelmäßig weise Entscheidungen treffen zu können. Wenn jedoch epistemisch signifikante Zustände gar nicht auf bewussten

dieser kompilatorische Versuch, die gegensätzlichen Intuitionen zu vereinen, gravierenden Einwänden ausgesetzt ist, Sharon Ryan, „Wisdom, Knowledge, and Rationality,” Acta Analytica 27, Nr. 2 (2012): 107. Ryan hofft, diese Einwände im Rahmen ihres aktuellen Vorschlags „The Deep Rationality Theory of Wisdom" (DRT) beseitigen zu können. Ich bezweifle jedoch, dass ihr das gelungen ist. Beispielsweise ist der von ihr verwendete Begriff der Rationalität äußerst unklar. In einem späteren Papier räumt Ryan ein, dass dieser Begriff einer genaueren Ausarbeitung bedarf. Unabhängig davon gibt es Probleme mit der Behauptung, dass alle Bedingungen von DRT notwendig und gemeinsam hinreichend sein sollen, Sharon Ryan, „A Deeper Defense of the Deep Rationality Theory of Wisdom: A Reply to Fileva and Tresan," Acta Analytica 32, Nr. 1 (2017). Vgl. zu dieser Kritik: Iskra Fileva und Jon Tresan, „Wisdom Beyond Rationality: A Reply to Ryan,” Acta Analytica 28 (2013) and Shane Ryan, „Wisdom: Understanding and the Good Life,” Acta Analytica 31, Nr. 3 (2016).

${ }^{4}$ In ähnlicher Form haben Fileva und Tresan (,Wisdom beyond rationality,” 234) betont, dass eine Person nur dann als weise zu betrachten ist, wenn sie in der Lage ist, im Hinblick auf geeignete Werte die richtigen Urteile zu fällen und entsprechende Entscheidungen treffen zu können. 
Entscheidungen beruhen, dann lässt sich eine solche Konzeption nicht auf die Zuschreibungen epistemisch signifikanter Einstellungen übertragen. Mit anderen Worten, die vorgeschlagene Strategie wäre von vornherein zum Scheitern verurteilt.

Es gibt eine sehr einfache Möglichkeit, diese grundlegende Skepsis zu entkräften. Wenn wir uns darauf einigen, dass epistemische Weisheit nur in solchen Kontexten der Zuschreibung kognitiver Zustände eine Rolle spielt, in denen es unproblematisch erscheint, von einem epistemischen Akteur zu behaupten, dass er oder sie bewusste Entscheidungen trifft, wäre das angeführte Problem vom Tisch. $^{5}$

Dagegen ließe sich einwenden, dass dieser Vorschlag eine bloße Verlegenheitslösung darstellt, da eine solche Einschränkung der Zuschreibung epistemischer Weisheit völlig willkürlich wäre.

Im Gegensatz dazu möchte ich im weiteren Verlauf dieser Arbeit dafür argumentieren, dass epistemische Weisheit tatsächlich vor allem in solchen $\mathrm{Zu}$ sammenhängen zu lokalisieren ist, in denen diejenige Person, der man das Prädikat „weise sein“ zuschreibt, bestimmte Entscheidungen zu treffen hat. Diese Tatsache erscheint mir weder willkürlich zu sein noch stellt der darauf aufbauende Vorschlag ein bloßes ad hoc Manöver dar. Der Ausgangspunkt dieser Annahme ist, dass epistemische Weisheit keine gewöhnliche „first-order" kognitive Einstellung ist; stattdessen argumentiere ich im Rahmen dieser Arbeit dafür, dass sich epistemische Weisheit in der Aktivität des weisen (kognitiven) Ratschlags verorten lässt. Diese Aktivität ist jedoch als ein komplexes interpersonales Geschehen zu begreifen, in dessen Rahmen ein testimonialer Informationstransfer zwischen einem Sprecher - der als kognitiver Ratgeber fungiert - und seinem Adressaten stattfindet. Innerhalb dieses interpersonalen Geschehens, so lautet meine zentrale These, nimmt die epistemische Position des Informationsgebers (Sprechers) eine unverwechselbare axiologische Qualität an. Dies wiederum führt mich zu der Annahme, dass der weise (kognitive) Ratschlag eine ganz besondere (kognitive) Aktivität ist, deren Erfolgsbedingungen mit keiner anderen Form der Zuschreibung eines epistemischen Zustands vergleichbar sind.

Im weiteren Verlauf dieser Arbeit wird es im Wesentlichen darum gehen, die eben gemachten Behauptungen zu plausibilisieren. Um etwas mehr Gripp zu bekommen, beginne ich meine Überlegungen mit einem konkreten Vorschlag zur begrifflichen Festlegung epistemischer Weisheit. Ausgangspunkt dieses Vorschlags ist die eben angesprochene These, dass Weisheit als erkenntnistheoretischer Begriff vor allem dann ins Spiel kommt, wenn man danach fragt, welche Anforderungen die epistemische Position erfüllen muss, in der sich eine

\footnotetext{
${ }^{5}$ Das betrifft natürlich nur das allgemeine Problem der epistemischen Übertragbarkeit des angesprochenen Ansatzes. Offen bleibt, wie weise Entscheidungen zu definieren sind und was es heißt, dass sich ein exzellenter (kognitiver) Ratgeber auf solche Entscheidungen stützen kann.
} 
Person befindet, sofern sie von anderen Personen als ein weiser kognitiver Ratgeber wahrgenommen wird. In Kurzform lautet mein Vorschlag wie folgt:

\section{Epistemische Weisheit (EW)}

Epistemische Weisheit ist ein metakognitiver Zustand in dem sich eine kognitiv überlegene Person befindet, sofern sie die Entscheidung zu treffen hat, was für die epistemischen Bedürfnisse des Adressaten ihrer kognitiven Aktivität langfristig gesehen das Beste ist, d. h. welche Informationen sie dem Adressaten ihrer testimonialen Aktivität übermitteln sollte. ${ }^{6}$

Wenn ich im Folgenden von epistemischer Weisheit spreche, beziehe ich mich auf diese Begriffsbestimmung. Die Kursivierung einzelner Ausdrücke signalisiert, dass sich mein Vorschlag auf eine Reihe von Unterbegriffen stützt, die für die Zuschreibung epistemischer Weisheit aus meiner Sicht von zentraler Bedeutung sind. In den nächsten Abschnitten werde ich versuchen, diese Unterbegriffe näher zu erläutern. Ich beginne damit, auf drei Besonderheiten der Zuschreibung epistemischer Weisheit aufmerksam zu machen. Epistemische Weisheit in der hier vertretenen Form unterscheidet sich von anderen Arten der Zuschreibung kognitiver Einstellungen sowohl in ontologischer, epistemischer als auch in axiologischer Hinsicht.

\section{BESONDERHEITEN DER ZUSCHREIBUNG EPISTEMISCHER WEISHEIT}

\subsection{Ontologische Besonderheit - Weisheit als metakognitiver Zustand}

Wenn wir einer Person eine epistemische Einstellung zuschreiben, verwenden wir für gewöhnlich Sätze der folgenden Form:

- S glaubt, dass p der Fall ist.

- S weiß, dass p der Fall ist.

- $\mathrm{S}$ weiß, warum $\mathrm{p}$ der Fall ist.

- S versteht, warum $\mathrm{p}$ der Fall ist.

Mit derartigen Sätzen wird zum Ausdruck gebracht, dass der Adressat der Zuschreibung - also diejenige Person, die S bezeichnet - eine von der Art des je-

\footnotetext{
${ }^{6}$ Einer der Gutachter dieser Arbeit hat angemerkt, dass nach dieser Charakterisierung von epistemischer Weisheit die Qualität der Entscheidung keine Rolle spielt, da es unerheblich sei, ob die Entscheidung selbst weise ist oder nicht. Das ist jedoch nicht der Fall. Eine Person erfüllt nur dann die angeführte Charakterisierung, wenn sie in der Lage ist, zu entscheiden, was für die epistemischen Bedürfnisse des Adressaten ihrer kognitiven Aktivität langfristig gesehen das Beste ist. Ich gehe davon aus - ohne das an dieser Stelle weiter rechtfertigen zu können -, dass es eine wesentliche Bedingung von weisen Entscheidungen ist, nur solche Handlungsoptionen oder kognitiven Alternativen zu wählen, die langfristig gesehen zu einer größtmöglichen Verbesserung der zu erwartenden Situation führen.
} 
weiligen Einstellungsverbs abhängige ,first-order“ Einstellung besitzt. Diese anerkannte linguistische Praxis der direkten Zuschreibung einer propositionalen Einstellung erweist sich jedoch im Fall von Weisheit als unangemessen. Denn wie die nachstehenden Versuche zeigen, wären die betreffenden Sätze in grammatikalischer Hinsicht nicht wohlgeformt:

- S weist, dass p der Fall ist.

- $\mathrm{S}$ weist, warum p der Fall ist.

Was heißt das für die Zuschreibung epistemischer Weisheit? Ganz offenkundig bedeutet dies nicht, dass Weisheit im epistemischen Zusammenhang überhaupt keine Rolle spielt. Auch wenn wir epistemische Weisheit nicht in Form einer „first-order" Einstellung direkt zuschreiben, können wir jemandem - im Sinne der oben angeführten Begriffsbestimmung - kognitive Ratschläge erteilen, was in einer Situation - im Hinblick auf die betreffenden epistemischen Bedürfnisse - langfristig gesehen das Beste ist. Die folgenden Sätze mögen vielleicht etwas ungewohnt erscheinen, ihrer Oberflächengrammatik ist jedoch in Takt:

- Es wäre das Beste (weise) für A, zu glauben, dass p.

- Es wäre das Beste (weise) für A, nicht nur zur glauben, dass p, sondern hinreichende Gründe zu haben, zu glauben, dass p.

- Es wäre das Beste (weise) für A, nicht nur hinreichende Gründe zu haben, zu glauben, dass $\mathrm{p}$, sondern zu wissen, dass $\mathrm{p}$.

- Es wäre das Beste (weise) für A, nicht nur zu wissen, dass p, sondern zu wissen, warum p.

- Es wäre das Beste (weise) für $\mathrm{A}$, nicht nur zu wissen, warum $\mathrm{p}$, sondern $\mathrm{zu}$ verstehen, warum p. $^{7}$

Die natürlichste Reaktion an dieser Stelle besteht meiner Ansicht nach darin, davon abzukommen epistemische Weisheit als eine Art „first-order" kognitive Einstellung zu betrachten. Die grammatikalische Oberflächenstruktur der betreffenden Sätze legt eine andere Deutung nahe. Nimmt man die Struktur ernst, kann man sagen, dass Personen, die solche Sätze zum Ausdruck bringen, in folgender Form eine , second-order “Einstellung zum Adressaten einnehmen: Ein Sprecher S teilt dem Adressaten A seiner kognitiven Aktivität mit, dass es weise wäre, sich in einer bestimmten ,first-order" Einstellung zu befinden oder diese zu erlangen.

\footnotetext{
${ }^{7}$ Im Rahmen der oben angeführten Charakterisierung ist eine Person in epistemischer Hinsicht weise, wenn sie in Bezug auf solche Zuschreibungen regelmäßig (epistemisch) erfolgreich ist. Derartige Personen sind in der Lage, anderen Menschen exzellente kognitive Ratschläge zu erteilen, in dem sie dem Adressaten ihrer kognitiven Aktivität mitteilen, was für ihn die bestmögliche epistemische Position ist, die er in einer bestimmten Situation erreichen kann. Das bedeutet jedoch nicht, dass der Adressat dadurch selbst eine Person ist, die in der angesprochenen Art und Weise epistemisch weise genannt werden kann.
} 
Dieser Gedanke gewinnt noch etwas mehr an Plausibilität, wenn wir uns Situationen des kognitiven Ratschlags genauer ansehen. Nehmen wir an, eine Person - die von mir um einen Rat gebeten wurde - tut mir kund, dass es das Beste für mich wäre, genauer zu verstehen, warum $\mathrm{p}$ und nicht $\mathrm{q}$ der Fall ist. Die Kundgabe des Ratschlags gibt mir keinen Hinweis darauf - zumindest keinen eindeutigen -, in welchem "first-order" Zustand sich die ratgebende Person befindet. Nichtsdestotrotz lässt sich dasjenige, was der Ratgeber mir mitzuteilen versucht, nur dann als Kundgabe eines Ratschlags interpretieren, wenn wir unterstellen, dass sich die ratgebende Person in einem mentalen Zustand befindet, der aus einer übergeordneten Metaperspektive heraus erfolgt und auf eine oder mehrere meiner ,first-order“ Einstellungen Bezug nimmt. Man kann daher sagen, dass Situationen des kognitiven Ratschlags paradigmatische Fälle einer Sprecher-Hörer-Interaktion sind, in denen sich der Sprecher - also die ratgebende Person - in einem metakognitiven Zustand befindet, der auf unterer Ebene auf eine oder mehrere aktuale bzw. mögliche „first-order" propositionale Einstellungen des Hörers Bezug nimmt. ${ }^{8}$

Mit der Annahme, dass der individuelle (mentale) Zustand, in dem sich eine Person befindet, wenn sie einen weisen (kognitiven) Ratschlag erteilt, ein genuin metakognitiver Zustand ist, sind aus meiner Sicht zwei Besonderheiten verbunden: Zum einen ist dieser metakognitive Zustand nicht notwendigerweise doxastischer Natur. Ein weiser Ratgeber kann dem Adressaten seiner kognitiven Aktivität zu verstehen geben, dass es für ihn langfristig gesehen das Beste ist, $\mathrm{p}$ zu glauben, ohne dass der Ratgeber selbst (vollständig) glaubt, dass p der Fall ist. Und ebenso gilt: Um einen weisen (kognitiven) Ratschlag erteilen zu können, muss der Sprecher selbst nicht glauben, dass es für ihn das Beste sei, $\mathrm{p}$ zu glauben. Die metakognitive Einstellung des weisen Ratschlags hat nicht in direkter, sondern nur in indirekter Form einen propositionalen Gehalt - nämlich nur

\footnotetext{
${ }^{8}$ Man könnte an dieser Stelle entgegnen, dass bei der Zuschreibung einer gerechtfertigten Überzeugung ein ähnliches Problem vorzuliegen scheint: Sätze der Form „S rechtfertigt, dass p“ sind offenkundig nicht wohlgeformt, wohingegen ein Satz wie „S ist gerechtfertigt, zu glauben, dass p“ grammatikalisch korrekt erscheint. Dennoch würde man im Fall einer gerechtfertigten Überzeugung nicht behaupten, dass es sich dabei um die Zuschreibung einer metakognitiven Einstellung handelt. Stattdessen lautete die gängige Auffassung: Bei der Rechtfertigung handelt es sich um hinzukommende normative Eigenschaft derselben „first-order“ doxastischen Einstellung. Und etwas Ähnliches könnte man auch in Bezug auf Weisheit behaupten. So wie eine Person gerechtfertigt sein kann, etwas zu glauben, kann sie eben auch ,weise sein“, etwas zu glauben, wobei mit „weise sein“ eine spezielle normative Eigenschaft des damit verbundenen doxastischen Zustands gemeint ist. Dem möchte ich jedoch widersprechen. Im Gegensatz zur Rechtfertigung scheint die Anwendung des Prädikats „weise sein“ in einer augenfälligen Form unabhängig von der jeweiligen Art des damit verbundenen „first-order“ Zustands zu sein. Das Prädikat „weise sein“ lässt sich - anders als im Fall der Rechtfertigung - nicht nur auf (rein) doxastische Einstellungen anwenden, sondern eben auch auf alle anderen „first-order“ kognitiven Zustände wie z. B. Wissen oder Verstehen. Im Vergleich dazu scheinen Sätze der folgenden Art „S ist gerechtfertigt, zu wissen, dass p“ eine Art von Kategorienfehler zu implizieren.
} 
dadurch, dass mit ihr implizit auf eine "first-order" kognitive Einstellung des Hörers Bezug genommen wird, die den Inhalt $\mathrm{p}$ hat. Aus diesem Grund kann ein weiser (kognitiver) Ratschlag selbst dann für den Adressaten der kognitiven Aktivität epistemisch wertvoll sein, wenn sich der Träger der metakognitiven Einstellung (d. h. die ratgebende Person) nicht im selben doxastischen Zustand, dass $p$, wie die ratsuchende Person befindet.

Die zweite Besonderheit betrifft die folgende Beobachtung: Das mit Abstand auffälligste Merkmal, das weise (kognitive) Ratschläge mit anderen Formen des Beratschlagens teilen, besteht darin, dass solche kognitiven Aktivitäten nur dann in epistemischer Hinsicht erfolgreich sein können - d. h. beim Adressaten eine epistemische Wirkung entfalten -, wenn sie vom Ratgeber artikuliert werden, d. h. explizit zum Ausdruck gebracht werden. Ein unartikulierter kognitiver Ratschlag - was immer es für eine Person bedeuten mag, in einem solchen Zustand zu sein -, hat ganz offenkundig keine epistemische Signifikanz. Mehr noch, von einem Ratschlag, der das Prädikat „weise sein“ wirklich verdient, ist zu verlangen, dass der Sprecher der kognitiven Aktivität in der Lage ist, die betreffende Information in angemessener Form zu artikulieren. Dazu reicht es nicht aus, dass die betreffende Information irgendwie übermittelt wird. Eine Person ist nur dann in der epistemischen Position, einer anderen Person einen weisen Ratschlag zu erteilen, wenn ihr dasjenige, was sie dem Adressaten ihrer testimonialen Aktivität übermittelt möchte, reflexiv zugänglich ist. ${ }^{9}$

Mit reflexiver Zugänglichkeit ist zweierlei gemeint: Einerseits müssen die Gründe für das Erfassen des Informationsgehalts dem Ratgeber in besonderer Form - nämlich internal - zugänglich sein. Und andererseits muss sich ein Informant, sofern er als weiser (kognitiver) Ratgeber eine epistemische Wirkung entfalten möchte, den normativen Verpflichtungen bewusst sein, die mit der Artikulation eines weisen (kognitiven) Ratschlags verbunden sind. Ein nur verlässlicher Informant, dem die Gründe für das korrekte Erfassen des zu übermittelnden Informationsgehalts selbst nicht zugänglich sind, oder dem die normativen Verpflichtungen seiner testimonialen Aktivität nicht klar sind - so die hier vertretene These -, ist nicht in der epistemischen Position, einen weisen (kognitiven) Ratschlag angemessen artikulieren zu können. ${ }^{10}$

\footnotetext{
${ }^{9}$ Im Alltagsgebrauch scheint es einen breiten Konsens zu geben, dass der mentale Zustand, in dem sich eine Person befindet, so sie in der Lage ist, weise Ratschläge zu erteilen, als „selbstreflexiv“ zu bezeichnen ist und bisweilen auch das Prädikat „kontemplativ“ verdient. Diese weitverbreitete These findet sich auch in der philosophisch-psychologischen Literatur zu diesem Thema an vielen Stellen wieder. Vgl. hierzu: Monika Ardelt, „Wisdom as Expert Knowledge System: A Critical Review of a Contemporary Operationalization of an Ancient Concept," Human Development 47, Nr. 5 (2004); Valerie Tiberius, The Reflective Life - Living Wisely within our Limits (New York: Oxford University Press, 2008); Judith Glück, Weisheit - Die 5 Prinzipien des gelingenden Lebens (München: Kösel-Verlag, 2016).

${ }^{10}$ Die Bedingungen der angemessenen Artikulation eines weisen Ratschlags lassen sich demnach in zwei Unterbedingungen aufteilen: (i) Schwache normative Bedingungen, die das korrekte Er-
} 
2.2. Epistemische Besonderheit

Weisheit als interpersonale Quelle der Erkenntnis

Ich habe eben argumentiert, dass man Weisheit als individuellen (mentalen) Zustand eines kognitiven Ratgebers nicht im Sinne einer ,first-order“" (kognitiven) Einstellung analysieren kann. Das Phänomen des weisen Ratschlags macht aber noch etwas anderes deutlich: Der weise Rategeber befindet sich in einem metakognitiven Zustand, dessen ,first-order“ Basis die kognitive Einstellung einer anderen Person ist. Diese andere Person steht - sofern es sich dabei um eine Situation des kognitiven Ratschlags handelt - in einer unmittelbaren Sprecher-Hörer-Interaktion zum Ratgeber. Der individuelle Zustand des Sprechers hat nur indirekt einen propositionalen Gehalt, nämlich dadurch, dass die metakognitive Einstellung des Sprechers auf eine „first-order“ kognitive Einstellung des Hörers Bezug nimmt.

An dieser Stelle drängt sich die folgende Frage auf: Was bedeutet es für die Charakterisierung der epistemischen Position des Sprechers, dass der individuelle (mentale) Zustand des weisen (kognitiven) Ratgebers nicht isoliert von der „first-order" kognitiven Einstellung des Hörers betrachtet werden kann. Welchen Einfluss hat es, dass weise (kognitive) Ratschläge - die, wie eben gesehen, artikuliert werden müssen - nur in Abhängigkeit von Sprecher-Hörer-Interaktionen eine epistemische Signifikanz erlangen. Um Verwechslungen auszuschließen, unterscheide ich zwischen dem individuellen (mentalen) Zustand und der epistemischen Position des weisen Rategebers wie folgt:

\section{Individueller Zustand des weisen Ratgebers}

Der individuelle (mentale) Zustand des weisen (kognitiven) Ratgebers ist durch die metakognitive Einstellung gekennzeichnet, mit der sich diejenige Person, die einen Ratschlag artikuliert (Sprecher) auf die „first-order“ kognitive Einstellung des Adressaten der testimonialen Aktivität (Hörer) bezieht.

\section{Epistemische Position des weisen Ratgebers}

Die epistemische Position des weisen (kognitiven) Ratgebers ist durch die interpersonale Rolle gekennzeichnet, in der sich diejenige Person befindet, die einen Ratschlag angemessen artikuliert (Sprecher), sofern der individuelle (mentale) Zustand dieser Person die Quelle der Erkenntnisbemühungen des Adressaten der testimonialen Aktivität (Hörer) ist, d. h. der Ratgeber als kognitiv überlegene Person vom Hörer anerkannt wird.

Gemäß dieser Unterscheidung ist es nicht - zumindest nicht allein - dem individuellen (metakognitiven) Zustand der ratgebenden Person zu verdanken, dass deren testimoniale Aktivität epistemische Signifikanz erlangt. Erst dadurch,

fassen eines Informationsgehalts als Gegenstand des weisen Ratschlags betreffen. (ii) Starke normative Bedingungen, die die normativen Verpflichtungen der Artikulation eines korrekt erfassten Informationsgehalts im Rahmen einer testimonialen Aktivität des weisen Ratschlags betreffen. 
dass der Sprecher vom Adressaten der testimonialen Aktivität als externe Quelle seiner Erkenntnisbemühungen anerkannt wird - indem der Hörer dem testimonialen Bericht des Sprechers sein Vertrauen schenkt -, gelangt der weise (kognitive) Rategeber in eine epistemisch wertvolle Position. Zur Illustration ein Beispiel:

Hans ist ein ambitionierter Schachspieler, der sich verbessern möchte. Sein langjähriger Spielpartner gibt ihm den Rat, noch mehr Wissen in Bezug auf verschiedene Spielvarianten zu erwerben. Der Trainer seiner Schachmannschaft bezweifelt das und rät Hans, seine Rechenkapazität zu verbessern. Nur so kann er die verschiedenen Spielvarianten angemessen verstehen. Hans ist unsicher, wem er vertrauen soll. In seiner Verzweiflung bittet er sein großes Idol, Magnus Carlson, einige Partien mit ihm zu spielen. Carlson rät ihm, sich mehr auf sein gutes intuitives Spielverständnis zu verlassen. Es wäre das Beste für ihn, wenn er stets auf intuitive Weise zu erschließen versucht, welches die richtige Spielvariante ist. Hans vertraut Carlson und wechselt daraufhin zum Blitzschach, wo er sein intuitives Spielverständnis viel besser einbringen kann. Danach gewinnt Hans viele große Turniere.

Diese Art der Lokalisierung epistemischer Weisheit ist mit einer Besonderheit verbunden: „First-order“ kognitive Einstellungen wie z. B. Überzeugungen können durch verschiedene individuell zugängliche Erkenntnisquellen (wie Sinneswahrnehmungen, Intuitionen, Erinnerungen, logisches Schließen usw.) hervorgebracht werden. Für epistemische Weisheit - die sich als metakognitive Einstellung in Sprecher-Hörer-Interaktionen des exzellenten kognitiven Ratschlags offenbart - gilt das ganz offenkundig nicht. Der weise (kognitive) Ratschlag stellt vielmehr eine interpersonale Form des Erkenntniserwerbs dar, nämlich eine solche, die vermittelt durch das Zeugnis anderer Personen (testimonial) zustande kommt.

Man könnte an dieser Stelle einwenden, dass diese Behauptung nicht nur im Fall von Weisheit, sondern generell irreführend ist, da das Zeugnis anderer Personen überhaupt keine eigenständige Erkenntnisquelle darstellt. Stattdessen sind testimonial erworbene Überzeugungen oder Wissen auf die Ausübung individueller Fähigkeiten zurückzuführen. ${ }^{11}$ Ich werde jedoch im weiteren Verlauf dafür argumentieren, dass man Testimonialität tatsächlich als einen interpersonalen Prozess begreifen sollte, der zwischen dem Sprecher eines testimonialen Berichts (der ratgebenden Person) und seinem Zuhörer (der ratsuchenden Person) vonstattengeht.

\footnotetext{
${ }^{11}$ Das würde man sagen, wem man im Sinne von David Hume eine reduktionistische Strategie der Erklärung testimonialem Wissens verfolgt. Demnach wird die Fähigkeit, Wissen aus zweiter Hand zu erlangen, auf die Operationen von anderen epistemischen Kapazitäten - wie z. B. die Kapazität, inferentielle Schlüsse zu ziehen - zurückgeführt. Aktuell wird eine solche Strategie unter anderem von Elisabeth Fricker, „Telling and Trusting: Reductionism and Anti-Reductionism in the Epistemology of Testimony," Mind 104 (1995) und Tomoji Shogenji, „A Defense of Reductionism about Testimonial Justification of Beliefs," Nous 40 (2006) verteidigt.
} 
Die Annahme, dass der weise (kognitive) Ratschlag eine genuin interpersonale Form der Informationsübermittlung ist, hat Auswirkungen auf die begriffliche Analyse epistemischer Weisheit. Denn wenn kognitive Ratschläge generell eine interpersonale Struktur besitzen, hängt die epistemische Position des weisen (kognitiven) Ratgebers nicht nur von den normativen Verpflichtungen ab, die ein solcher Ratgeber bezüglich der Angemessenheit seiner metakognitiven Einstellung zu berücksichtigen hat, sondern auch von den „first-order“ kognitiven Einstellungen des Adressaten der testimonialen Aktivität, d.h. die epistemische Position hängt davon ab, dass der Adressat die ratgebende Person als geeignete Quelle seiner epistemischen Bemühungen anerkennt, indem er ihr - als einer Person, die ihm kognitiv überlegen ist - sein Vertrauen schenkt.

Zusammenfassend lassen sich an dieser Stelle zwei zentrale Aspekte hervorheben: Die testimoniale Aktivität des weisen (kognitiven) Ratschlags ist einerseits als ein individueller metakognitiver Zustand der Person des Ratgebers zu analysieren. Dieser metakognitive Zustand - der sich direkt auf die „first-order“" (kognitive) Einstellung des Adressaten der testimonialen Aktivität bezieht - ist selbst nicht notwendigerweise doxastischer Natur. Demgegenüber ist die epistemische Position des weisen (kognitiven) Ratgebers - sofern der Adressat der testimonialen Aktivität den metakognitiven Zustand des Ratgebers als Quelle seiner aktualen Erkenntnisbemühungen betrachtet - als ein interpersonaler Zustand zu charakterisieren. Im Gegensatz zum individuellen metakognitiven Zustand beruht die epistemische Position des weisen (kognitiven) Ratgebers auf normativen Verpflichtungen, ${ }^{12}$ die sich aus den epistemischen Erfolgsbedingungen der Sprecher-Hörer-Interaktion ergeben. Letzteres macht deutlich, dass sich eine angemessene Charakterisierung der epistemischen Position des weisen (kognitiven) Ratgebers nicht am Prinzip der kognitiven Autonomie festmacht.

\subsection{Axiologische Besonderheit}

Weisheit als eine interdependente kognitive Leistung

Wie die vorgeschlagene Begriffsdefinition verdeutlicht, verdient ein kognitiver Ratschlag nur dann das Prädikat ,weise zu sein“, wenn er auf der Grundlage der Entscheidung des Sprechers erfolgt, nur solche Informationen zu übermitteln, die für die epistemischen Bedürfnisse des Adressaten langfristig gesehen das Beste sind. Die Tatsache, dass ein weiser (kognitiver) Ratgeber eine Entscheidung treffen muss, welche Informationen er dem Adressaten zukommen lässt und welche nicht, macht epistemische Weisheit in axiologischer Hinsicht zu etwas Besonderem. Der Grund hierfür ist der Folgende: Wie auch immer man

\footnotetext{
12 Worin diese bipolaren normativen Verpflichtungen bestehen, wird an späterer Stelle (siehe Abschnitt 4, insbesondere Fn. 19) noch deutlich werden.
} 
epistemische Werte definiert, ${ }^{13}$ eine solche Definition sollte im Einklang damit stehen, dass der Wert einer kognitiven Aktivität nicht allein vom epistemischen Erfolg, sondern auch vom kognitiven Einsatz (Leistung oder Anstrengung) abhängig ist, der dafür erbracht werden musste. ${ }^{14}$

Meine These hierzu lautet, dass der weise (kognitive) Ratschlag aufgrund des interpersonalen Zusammenhangs der Sprecher-Hörer-Interaktion eine komplexe interdependente kognitive Leistung ist, die sich in dieser Form weder bei der Zuschreibung von wahren Meinungen, Wissen oder Verstehen wiederfindet. In individueller Perspektive unterscheidet sich die testimoniale Aktivität des weisen Ratschlags von anderen „first-order" kognitiven Aktivitäten wie folgt: Zum einen muss der weise (kognitive) Ratgeber einschätzen können - quasi in simultaner Betrachtung der epistemischen Bedürfnisse des Hörers -, was es im Lichte der Erkenntnisbemühungen des Adressaten seiner kognitiven Aktivität bedeutet, epistemisch erfolgreich zu sein. Zum anderen muss der weise (kognitive) Ratgeber zwischen verschiedenen kognitiven Alternativen - die mit Blick auf einen kurzfristigen kognitiven Erfolg möglicherweise gleichwertig erscheinen - eine korrekte Entscheidung treffen, d. h. er muss entscheiden, was langfristig gesehen unter Berücksichtigung gleichwertiger kognitiver Alternativen für den Adressaten (epistemisch gesehen) das Beste ist.

Anhand dieses besonderen entscheidungstheoretischen Aspekts lässt sich verdeutlichen, inwiefern der weise (kognitive) Ratschlag eine distinkte kognitive Leistung darstellt, die sich von der Leistung, die andere Arten von kognitiven Aktivitäten implizieren, signifikant unterscheidet. Betrachten wir dazu die folgende Abgrenzung:

\footnotetext{
${ }^{13}$ Gemäß der von mir vertretenen Auffassung sind epistemische Werte im Sinne einer teleologischen Konzeption als relative Werte - nämlich relativ zur epistemischen Art des Ziels der Untersuchung - zu analysieren. Ein Überblick über die verschiedenen Ansätze der Erklärung epistemischer Werte wird in Pedro Schmechtig, „Epistemische Werte,” in Handbuch Erkenntnistheorie, hg. Martin Grajner und Guido Melchior (Stuttgart: Metzler, im Druck 2019) gegeben.

${ }^{14}$ An dieser Stelle sind zwei Fragen zu unterscheiden: (i) Sind kognitive Leistungen als Erfolg aufgrund von Fähigkeiten zu definieren? Diese Frage betrifft die Natur der Leistung. (ii) Besitzen kognitive Leistungen einen (wie zumeist behauptet wird) finalen Wert? Letzteres betrifft die Art des Werts der Leistung. Meiner Ansicht nach ist der Wert einer Leistung ein bedingter Wert, da er selbst vom Wert des Ziels (Ergebnis) der Leistung abhängt. Meine Erklärungsstrategie ist es, Leistungen allgemein als ein Wertganzes zu betrachten. Demnach beinhalten Leistungen (generell) bestimmte inhärente Eigenschaften (wie die Überwindung von Schwierigkeiten und kompetente Verursachung) und ein externes Ziel. Der Wert der Leistung ist aber nicht einfach die Summe der Teilwerte (Wert der inhärenten Eigenschaften plus Wert des externen Ziels). Vielmehr schätzen wir Leistungen als organische Einheit bestehend aus »Schwierigkeiten, kompetente Verursachung, Ziel«. Bei kognitiven Leistungen ist dieses Ziel epistemischer Natur (z. B. Wissen, wahre Meinung, oder Verstehen).
} 


\section{Epistemische Weisheit als distinkte individuelle Leistung}

In Situationen in denen es zwei gleichwertige epistemische Alternativen $\mathrm{p}$ und $\mathrm{p}^{*}$ gibt, muss ein weiser (kognitiver) Ratgeber, um epistemisch angemessen agieren zu können, eine Entscheidung treffen, ob p oder $\mathrm{p}^{*}$ für die epistemischen Bedürfnisse derjenigen Person, an die sein Ratschlag adressiert ist, langfristig gesehen das Beste ist. ${ }^{15}$

Etwas Ähnliches gilt weder für Wissen noch für Verstehen: In einer Situation, in der $\mathrm{S}$ weiß, dass $\mathrm{p}$, und gleichzeitig weiß, dass $\mathrm{p}^{*}$, muss $\mathrm{S}-$ um epistemisch korrekt agieren zu können - nicht entscheiden, ob p oder $\mathrm{p}^{*} \mathrm{zu}$ wissen in epistemischer Hinsicht das Beste ist. ${ }^{16}$ Und ebenso gilt: In einer Situation in der S versteht , $\mathrm{q}$ aufgrund von $\mathrm{p}^{\prime}$ und gleichzeitig versteht , $\mathrm{q}$ aufgrund von $\mathrm{p}^{*}$ “, muss $\mathrm{S}$ - um epistemisch korrekt agieren zu können - nicht entscheiden können, ob $\mathrm{p}$ oder p* in epistemischer Hinsicht das Beste ist, d.h. ob p oder p* eine bessere oder tiefere Erklärung für q liefert. ${ }^{17}$

Gemäß dieser Abgrenzung muss ein weiser (kognitiver) Ratgeber, um epistemischen Erfolg haben zu können, eine individuelle kognitive Leistung erbringen, die bei anderen Arten der korrekten Zuschreibung eines epistemisch signifikanten Zustands nicht notwendig erscheint. Diese Besonderheit liefert den ersten Teil der Stützung meiner Behauptung, dass die individuelle (metakognitive) Einstellung des weisen (kognitiven) Ratschlags eine unverwechselbare axiologische Qualität besitzt.

Das ist aber noch nicht alles. Der eben angesprochene (individuelle) Aspekt ist mit einer weiteren Besonderheit der Zuschreibung epistemischer Weisheit verbunden. Gemäß der angeführten Begriffsbestimmung ist der weise (kognitive) Ratschlag eine testimoniale Aktivität, deren epistemischer Erfolg davon abhängig ist, ob die individuelle Leistung des Ratgebers vom Adressaten der testimonialen Aktivität als solche anerkannt wird, d. h. der Adressat dem Rat-

\footnotetext{
${ }^{15}$ Die Entscheidung, ob p oder p* langfristig gesehen die bestmögliche epistemische Alternative ist, muss natürlich vom Ratgeber in Relation zur Art des jeweiligen kognitiven „first-order“ $\mathrm{Zu}$ stands des Adressaten (Überzeugung, Wissen, Verstehen usw.) getroffen werden.

${ }^{16}$ Dasselbe trifft meiner Ansicht nach auch für praktische Formen des Wissens zu.

${ }^{17}$ Demnach kann epistemische Weisheit zwar so etwas wie „tiefes“ interrogatives Verstehen beinhalten, ist aber selbst nicht mit einem solchen kognitiven Zustand identisch. Das ist ein Punkt, der meiner Ansicht nach in der Literatur oft übersehen wird. Der Grund hierfür ist, dass epistemische Rationalität im Zusammenhang mit weisen (kognitiven) Ratschlägen eine metakognitive Einstellung ist. Selbst wenn man annimmt, dass diese metakognitive Einstellung nur dann epistemisch korrekt ist, sofern sie auf ein „tiefes“ Verstehen abzielt, werden im Zusammenhang mit weisen kognitiven Ratschlägen höhere Anforderungen an diese Art des epistemischen Verstehens gestellt. Für den weisen (kognitiven) Ratgeber reicht es nicht aus, sich in irgendeinem epistemisch angemessenen „first-order" Zustand des Verstehens zu befinden; vielmehr muss der weise (kognitive) Ratgeber entscheiden können, welcher „first-order“ Zustand des Verstehens im Hinblick auf gleichwertige Alternativen für die epistemischen Bedürfnisse der ratsuchenden Person der Beste wäre.
} 
geber - als einer Person, die ihm kognitiv überlegen ist - sein Vertrauen entgegen bringt. Ein kognitiver Ratschlag mag noch so exzellent sein, wenn der Träger der metakognitiven Einstellung nicht in der epistemischen Position ist, vom Adressaten des kognitiven Ratschlags als Quelle seiner Erkenntnisbemühungen akzeptiert zu werden, wird die testimoniale Aktivität - ganz gleich von welcher Art die individuelle Anstrengung des Ratgebers ist - erfolglos sein, d. h. sie wird in epistemischer Hinsicht keine normative Kraft entfalten.

Gemäß dieser zweiten Besonderheit hängt der epistemische Wert der testimonialen Aktivität des weisen (kognitiven) Ratschlags nicht nur von der individuellen (kognitiven) Leistung des Ratgebers, sondern auch von der Frage ab, unter welchen Bedingungen der Adressat des kognitiven Ratschlags bereit ist, dem potentiellen Träger einer testimonialen Aktivität zu vertrauen. Aus diesem Grund spreche ich von einer interdependenten kognitiven Leistung. Das grundlegende Problem an dieser Stelle ist folgendes: Ein Ratgeber kann in epistemischer Hinsicht ein guter Informant sein, nämlich dann, wenn wir uns darauf verlassen können, dass er nur an wahren Dingen interessiert ist und auf dieser Grundlage entscheidet, welche Informationen für den Adressaten das Beste sind. Und dennoch kann es sein, dass der kognitive Erfolg des Ratschlags ausbleibt, weil der Adressat der testimonialen Aktivität im Hinblick auf das Ziel seiner Erkenntnisbemühungen kein Vertrauen in den Sprecher hat, d.h. den Informanten nicht als Quelle des Erreichens seiner Erkenntnisinteressen akzeptiert. Doch welchen Grund gibt es dafür, zu behaupten, dass ein verlässlicher (guter) Informant nicht zwangsläufig ein weiser (kognitiver) Ratgeber ist?

In der philosophischen Literatur zum Begriff des Vertrauens ${ }^{18}$ wird allgemein anerkannt, dass Verlässlichkeit und Vertrauen nicht dasselbe sind. Man kann sich auf eine Person verlassen, selbst wenn es nicht gerechtfertigt ist, ihr zu vertrauen. Jemandem zu vertrauen - in welcher Hinsicht auch immer - beinhaltet mehr als nur die Tatsache, dass man sich auf sie verlassen kann. Angewandt auf den testimonialen Zusammenhang bedeutet das: Einer Person als weisen (kognitiven) Ratgeber zu vertrauen, beinhaltet mehr als die Annahme, dass diese Person ein verlässlicher (guter) Informant ist, d. h. immer nur wahre Dinge berichtet. Ein guter Informant mag ein verlässlicher epistemischer Akteur sein; das allein macht ihn aber noch nicht zu einer kognitiv überlegenen Person, der ein Adressat sein Vertrauen schenkt, wenn es um weise (kognitive) Ratschläge geht. Im Fall des weisen (kognitiven) Ratgebers kommt eine zusätzliche normative Komponente ins Spiel: Der Adressat muss einen guten Informanten als Quelle seiner epistemischen Bedürfnisse anerkennen.

\footnotetext{
${ }^{18}$ Vgl. zu dieser Diskussion: Annette Baier, „Trust and Antitrust,” Ethics 96, Nr. 2 (1984); Paul Faulkner, „On Telling and Trusting,” Mind 116 (2007); Karen Frost-Arnold, „The Cognitive Attitude of Rational Trust,” Synthese 191 (2012); Richard Holton, „Deciding to Trust, Coming to Believe," Australasian Journal of Philosophy 72, Nr. 1 (1994); Benjamin McMyler, Testimony, Trust, \& Authority (New York: Oxford University Press, 2011).
} 
Die Berücksichtigung dieser normativen Komponente hat Auswirkungen auf die axiologische Analyse: Selbst wenn wir annehmen würden - entgegen der vorangegangen Überlegung -, dass im Hinblick auf die individuelle kognitive Leistung kein Unterschied besteht, ob wir es mit einem weisen (kognitiven) Ratgeber, einem Wissenden oder einem Verstehenden zu tun haben, sorgt diese zusätzliche Komponente dafür, dass die Analyse des epistemischen Werts der Weisheit nicht mit der von anderen (epistemischen) Werten wie Wissen oder Verstehen vergleichbar ist. Mit der Frage der Anerkennung einer kognitiv überlegenen Person kommt ein interpersonaler Aspekt der Bewertung des epistemischen Erfolgs kognitiver Aktivität ins Spiel, der über die Frage hinausweist, ob es in epistemischer Hinsicht angemessen ist, die individuelle kognitive Aktivität eines (guten) Informanten wertzuschätzen oder nicht.

An dieser Stelle ist jedoch zu betonen, dass die eben gemachten Behauptungen nicht unumstritten sind. Insbesondere lässt sich natürlich fragen, ob es wirklich zutreffend ist, im Zusammenhang mit epistemischer Weisheit von einer testimonialen Quelle zu sprechen, die im Sinne einer Sprecher-Hörer-Interaktion als ein interpersonales Geschehen zu begreifen ist. Betrachtet man die in der Erkenntnistheorie gängige Diskussion zwischen reduktionistischen und antireduktionistischen Modellen der Erklärung testimonialen Wissens, scheint es trotz aller Unterschiede beider Herangehensweisen eine fundamentale Gemeinsamkeit zu geben, nämlich die Annahme, dass testimoniales Wissen mit dem Prinzip der kognitiven Autonomie vereinbar sein sollte. Die Anwendung eines solchen Prinzips auf den vorliegenden Zusammenhang scheint zu besagen, man müsse den Wert des weisen (kognitiven) Ratschlags - zumindest dann, wenn es um die epistemische Dimension dieses Werts geht - anhand der individuellen kognitiven Leistung der ratgebenden Person bestimmen. Es ist zu bezweifeln, dass man testimoniale Aktivitäten in Abhängigkeit zu irgendwelchen SprecherHörer-Interaktionen analysieren sollte. Wenn man epistemische Weisheit in Analogie zur testimonialen Analyse von (propositionalen) Wissen als eine individuell zugängliche Erkenntnisquelle begreift, gibt es überhaupt keinen Anlass an einer derartigen These festzuhalten.

Derartige Einwände offenbaren ein Problem von allgemeiner Art. Der vorliegende Ansatz geht davon aus, dass epistemische Weisheit eine testimoniale Aktivität ist, die aufgrund ihrer besonderen interpersonalen Struktur einen unverwechselbaren epistemischen Wert besitzt. Traditionelle Erklärungen testimonialer Aktivitäten lehnen jedoch ein solches interpersonales Modell ab, da es das Prinzip der kognitiven Autonomie verletzt. Die oben angeführte Unterscheidung zwischen dem individuellen (metakognitiven) Zustand und der epistemischen Position des weisen (kognitiven) Ratgebers macht hingegen deutlich, dass das nur teilweise der Fall ist. Im nächsten Abschnitt versuche ich daher zu zeigen, dass eine solche (teilweise) Einschränkung überhaupt kein Nachteil sein muss. Das wesentlich größere Problem umgekehrt darin besteht, dass traditionelle 
Erklärungsversuche nicht in der Lage sind, den besonderen ,,secondhand“ Charakter erklären zu können, den eine testimoniale Aktivität als eigenständige Form der Quelle unserer Erkenntnisbemühungen auszeichnet. Dieses Problem scheint mir im Fall des weisen (kognitiven) Ratschlags besonders kontraintuitiv zu sein. Aus diesem Grund plädiere ich im Folgenden für eine Abkehr von der traditionellen Sichtweise.

\section{DIE INTERPERSONALE ERKLÄRUNG TESTIMONIALER RECHTFERTIGUNG}

Ist Testimonialität ein interpersonales Phänomen? Traditionelle Ansätze der Erklärung testimonialen Wissens haben diese Frage verneint. Der reduktionistische Ansatz geht davon aus, dass Testimonialität generell nicht als eigenständige Erkenntnisquelle zu betrachten ist. Dass ein Hörer $\mathrm{H}$ aufgrund eines Berichts des Sprechers $\mathrm{S}$ glaubt oder weiß, dass $\mathrm{p}$, verdankt $\mathrm{H}$ nicht der testimonialen Aktivität des Sprechers, sondern seiner individuellen (inferentiellen) Fähigkeit,

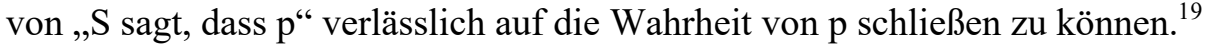

Der klassische, auf Thomas Reid zurückgehende anti-reduktionistische Ansatz behauptet hingegen, dass Testimonialität eine eigenständige Erkenntnisquelle ist. ${ }^{20}$ Doch analog zur Sinneswahrnehmung soll es sich dabei um eine individuell zugängliche Quelle handeln. Infolgedessen bedarf es keiner eigenständigen Begründung - die über die individuelle Perspektive der Rechtfertigung hinausgeht -, warum $\mathrm{H}$ dem Sprecher $\mathrm{S}$ als testimonialer Quelle seiner Erkenntnisbemühungen das Vertrauen schenkt.

Im Folgenden versuche ich zu zeigen, dass die Berücksichtigung epistemischer Weisheit dazu führt, weder die eine noch die andere Position zu vertreten, sondern einen Mittelweg einzuschlagen, der die Fallstricke beider (traditioneller) Ansätze vermeidet.

Meiner Ansicht nach offenbart das Phänomen des weisen (kognitiven) Ratschlags, dass Testimonialität tatsächlich eine eigenständige Quelle ist, d. h. nicht auf die Fähigkeiten des Hörers als Quelle der Erkenntnisbemühungen reduziert werden kann (entgegen des reduktionistischen Ansatzes). In keinem anderen Fall einer testimonialen Aktivität wird deutlicher, dass der Sprecher eigenverantwortlich etwas tun muss. Erinnern wir uns: Der weise (kognitive) Ratgeber muss entscheiden, welche Informationen er dem Hörer - im Sinne der langfristigen Erfüllbarkeit seiner Erkenntnisbemühungen - übermitteln sollte. Dieser

\footnotetext{
${ }^{19}$ Vgl. hierzu genauer: McMyler, „Testimony,” 50.

${ }^{20}$ Nach Reid beruht die Tatsache, dass wir auf testimoniale Weise Informationen erlangen können, auf einer basalen (kognitiven) Fähigkeit, deren Ausübung analog zur Wahrnehmung auf noninferentiellen Zusammenhängen beruht, Thomas Reid, An Inquiry into the Human Mind (University Park, Pennsylvania: Penn State University Press, 1997), 200.
} 
Bestandteil der testimonialen Aktivität lässt sich nicht auf Operationen der epistemischen Kapazität des Hörers reduzieren.

Andererseits bedarf es aber auch einer Rechtfertigung, warum der Adressat eines weisen (kognitiven) Ratschlags dem Sprecher vertraut, d. h. der Hörer Grund dazu hat, den Sprecher als testimoniale Autorität anzuerkennen (entgegen des anti-reduktionistischen Ansatzes). Wie wir gesehen haben ist ein guter Informant nicht automatisch ein kognitiver Ratgeber, der in epistemischer Hinsicht weise genannt werden kann.

Angesichts der im vorangegangenen Abschnitt herausgestellten Besonderheiten von epistemischer Weisheit lautet mein Vorschlag wie folgt: Eine angemessene Erklärung der testimonialen Aktivität des weisen (kognitiven) Ratschlags ist nur dann möglich, wenn man einen Mittelweg einschlägt, indem man versucht, den speziellen interpersonalen Charakter dieser Erkenntnisquelle zu erfassen. Demnach ist der weise (kognitive) Ratschlag eine eigenständige Quelle, die jedoch im Hinblick auf die Anerkennung der epistemischen Position des weisen (kognitiven) Ratgebers auf der Seite des Hörers einer Rechtfertigung bedarf, d. h. der Hörer muss Grund dazu haben, einen verlässlichen (guten) Informanten als weisen (kognitiven) Ratgeber - im Sinne einer testimonialen Quelle seiner epistemischen Bedürfnisse - vertrauen zu können. Die zu klärende Frage lautet dann: Wie lässt sich im Rahmen eines interpersonalen Modells testimonialer Rechtfertigung erklären, dass der Adressat eines weisen (kognitiven) Ratschlags Grund dazu hat, dem Sprecher des testimonialen Berichts als kognitiven Ratgeber zu vertrauen?

Autoren wie Richard Moran (2005), Edward Hinchman (2005, 2014), Paul Faulkner (2007, 2011), Benjamin McMyler (2011), Linda Zagzebski (2012) haben in den letzten Jahren auf unterschiedliche Weise hervorgehoben, dass die traditionelle Debatte zu stark auf den Begriff der individuellen Rechtfertigung in Verbindung mit testimonialem Wissen eingeengt ist. Was wir stattdessen benötigen, ist ein Begriff von testimonialer Rechtfertigung, der den besonderen ,secondhand" Charakter dieser Art der Erkenntnisquelle zum Vorschein bringt. Was ist damit gemeint? McMyler (2011) hat diesen Gedanken sehr klar wie folgt formuliert:

\section{„Secondhand“ Charakter testimonialen Wissens}

An item of knowledge is testimonial just in case it is scondhand in the demanding sense of being justified by appeal to the authority of the speaker (Hervorhebung PSch $)^{21}$

\footnotetext{
${ }^{21}$ Benjamin McMyler, Testimony, Trust, \& Authority (Oxford University Press: 2011), 59. Ich verwende in diesem Zusammenhang den engen Begriff der testimonialen Autorität um weite Fälle auszuschließend, in denen man auch dann von einer epistemische Autorität spricht, wenn kein testimonialer Sprechakt oder die Artikulation irgendeines Ratschlags vorliegt. Die Unterscheidung von epistemischen Autoritäten in einem weiten Sinne und dem enger gefassten Begriff der testi-
} 
Es mag unterschiedliche Ansichten dazu geben, ob Wissen in diesem robusten Sinne eine testimoniale Quelle der Erkenntnis ist. Wenn wir jedoch den „secondhand" Charakter dieser Quelle wirklich ernst nehmen - und ich meine, wir sollten das tun -, dann ist klar, dass die Rechtfertigung einer kognitiven Einstellung, die auf testimoniale Weise zustande kommt, in einer Art und Weise durch den Bericht des Sprechers vermittelt sein muss, wie das bei einer Rechtfertigung durch Sinneswahrnehmungen oder durch Schlussfolgerungen nicht gegeben ist. Dies wird besonders deutlich, wenn man sich genauer anschaut, was es bedeutet, unter signifikanter Berufung auf die testimoniale Autorität des Sprechers in einer kognitiven Einstellung gerechtfertigt zu sein.

Die Idee einer ,signifikanten Berufung“ auf die testimoniale Autorität des Sprechers lässt sich im Rahmen des nachstehenden Modells am besten erklären: ${ }^{22}$

\section{„Buck-Passing“ - Erklärung testimonialer Rechtfertigung \\ Wenn ein Höher $\mathrm{H}$ auf der Basis eines testimonialen Berichts des Sprechers S zu einer kognitiven Einstellung, dass p, gelangt und $\mathrm{H}$ kann $\mathrm{S}$ als testimonialer Au- torität vertrauen, dann erwirbt $\mathrm{H}$ das epistemische Recht, Einwände gegenüber $\mathrm{H}^{\prime}$ 's kognitiver Einstellung, die von einer dritten Partei vorgebracht werden, zu- rückzuweisen, indem $\mathrm{H}$ die Notwendigkeit der Rechtfertigung seiner kognitiver Einstellung an S weiterreicht.}

Diese Erklärung macht deutlich, warum ein Modell testimonialer Autorität, das den ,secondhand“ Charakter dieser Quelle ernst nimmt, mit keinem der beiden traditionellen Ansätze vereinbar ist. Der reduktionistische Ansatz begeht den Fehler, den secondhand Charakter der testimonialen Quelle eliminieren zu wollen, da der Hörer $\mathrm{H}$ ganz offenkundig nicht unter einer signifikanten Berufung auf den Sprecher S zu einer Rechtfertigung seiner kognitiven Einstellung gelangt. Im Vergleich dazu scheint der anti-reduktionistische Ansatz den ,secondhand" Charakter zwar irgendwie anzuerkennen; nichtsdestotrotz lässt dieser Ansatz im Unklaren, inwiefern S's testimoniale Autorität eine substantielle Rolle für die Rechtfertigung der kognitiven Einstellung des Hörers spielt. Die „Buck-Passing“-Erklärung macht hingegen diese substantielle Rolle kenntlich: In diesem Modell hat der Hörer H nur deshalb eine epistemisch korrekte Einstellung, weil sich $\mathrm{H}$ in der Rechtfertigung seiner Einstellung unmittelbar $-\mathrm{d}$. h. in signifikanter Form - auf die testimoniale Autorität des Sprechers stützt. Gemäß dieser Erklärung beruht das Vertrauen, das der Hörer einer testimonialen Autorität entgegen bringt, nicht auf der individuellen Kompetenz des Hörers, bestimmte Schlüsse zu ziehen. Vielmehr basiert dieses Vertrauen auf der Anerkennung

monialen Autorität wird unter anderen in Linda Zagzebski, Epistemic Authority - A Theory of Trust, Authority, and Autonomy in Belief (New York: Oxford University Press 2012), 119 ff. diskutiert.

${ }^{22}$ Vgl. ebenso: McMyler, „Testimony.” 
der sozialen Funktion des Sprechers, für den Hörer eine geeignete „secondhand“ Quelle seiner epistemischen Bedürfnisse sein zu können. Diese Anerkennung der sozialen Funktion des Sprechers erlaubt es dem Hörer, die epistemische Pflicht der Rechtfertigung seiner kognitiven Einstellung an den Sprecher weiterreichen zu dürfen. ${ }^{23}$

In der eingangs eingeführten Begriffsbestimmung wurde deutlich gemacht, dass epistemische Weisheit ein metakognitiver Zustand ist, in dem sich eine kognitiv überlegene Person befindet. ${ }^{24}$ Es stellt sich jedoch die Frage, von welcher Art diese kognitive Überlegenheit ist? Die eben vorgeschlagene interpersonale Erklärung testimonialer Rechtfertigung geht davon aus, dass es sich bei kognitiv überlegenen Personen um testimoniale Autoritäten handelt. Demgegenüber kann jedoch der folgende Einwand erhoben werden: Es ist zu bezweifeln, dass weise Personen - um als kognitive Ratgeber bestimmte Entscheidungen treffen zu können - die interpersonale Rolle einer testimonialen Autorität ein-

\footnotetext{
${ }^{23}$ Traditionelle Ansätze erfassen nicht die bipolaren normativen Abhängigkeiten, die zwischen der Verantwortung einer testimonialen Autorität als „,secondhand“ Quelle und dem komplementären Vertrauen besteht, dass der Adressat der testimonialen Aktivität in diese Quelle setzt. Der vorliegende Ansatz trägt diesen Abhängigkeiten hingegen Rechnung. Nach McMyler, „Testimony,” Kapitel 4) muss dazu zwischen einer „third-personal“ und „,second-personal“ Zuschreibung des Vertrauens unterschieden werden. Eine Zuschreibung der Form „Peter vertraut Mary, dass sie ihn heiraten wird“ ist „second-personal“ da es bei der epistemischen Korrektheit dieser Zuschreibung nicht darum geht, herauszufinden, ob eine bestimmte Tatsache - auf die sich Peters individuelle Einstellung bezieht - wahr oder falsch ist. Anders als im ,third-personal“ Fall ist eine korrekte Zuschreibung von Vertrauen, das man ,in eine Person“ setzt, an die Erfüllung bipolarer normativer Verpflichtungen gebunden. Damit solche Zuschreibungen korrekt sind, müssen drei Dinge erfüllt sein: (i) Peter muss Mary als „second-personal“ Autorität anerkennen. (ii) Im Hinblick auf Peters Vertrauen muss sich Mary der Verantwortung bewusst sein, die sie für Peter hat, mit dem was sie tut. (iii) Und wenn Mary nicht das tut, was Peter aufgrund seines Vertrauens von ihr erwartet, muss klar sein, dass Peter die Berechtigung hat, seine Missbilligung gegenüber Mary zum Ausdruck zu bringen.
}

Es ist jedoch zu betonen, dass reduktionistische Ansätze trotz aller Kritik eine berechtigte Forderung erheben: Wenn es im Gegensatz zur Wahrnehmung keine angeborene Fähigkeit ist, einer testimonialen Autorität zu vertrauen, bedarf es einer Erklärung der Bedingungen, unter denen die (kognitive) Einstellung des Vertrauens gerechtfertigt ist. Der „Buck-Passing“ Ansatz liefert zwar eine allgemeine Erklärung wie ein testimonialer Transfer interpersonal funktioniert. Er lässt jedoch offen, unter welchen Voraussetzungen man im Rahmen einer Sprecher-HörerInteraktion individuell gerechtfertigt ist, dem Sprecher $\mathrm{S}$ zu vertrauen, d. h. er sagt nichts darüber, was $\mathrm{H}$ dazu bringt, ein Sprecher S als testimoniale Autorität zu akzeptieren. Innerhalb des interpersonalen Modells muss daher zusätzlich geklärt werden, was es im Zusammenhang mit der Kundgabe eines testimonialen Berichts bedeutet, einer testimonialen Autorität das Vertrauen zu schenken. Eine Beantwortung dieser Frage würde jedoch den Rahmen dieser Arbeit sprengen; sie muss daher anderenorts erfolgen.

${ }^{24}$ Die Annahme der kognitiven Überlegenheit ist darin begründet, dass Ratschläge im Allgemeinen nur dann sinnvoll erscheinen, wenn zwischen Sprecher und Höher ein asymmetrisches Verhältnis im Hinblick auf den Zugang und die kognitive Qualität der Verarbeitung von Informationen besteht. 
nehmen müssen. Vielmehr ist es so, dass sich weise Personen im Prozess des kognitiven Ratschlags auf genau denselben Typ von individuellen Fertigkeiten verlassen können, der auch der Entscheidungsfindung von Experten zugrunde liegt. Infolgedessen ist ein solcher interpersonaler Ansatz abzulehnen, denn es scheint nicht so zu sein, dass die Art von kognitiver Überlegenheit, die weise Personen auszeichnet - wenn sie als kognitive Ratgeber in Erscheinung treten -, etwas mit der vermeintlich interpersonalen Rolle von testimonialen Autoritäten zu tun hat.

Möchte man an dem vorgeschlagenen interpersonalen Ansatz festhalten, muss näher begründet werden, warum es notwendig ist, dass weise (kognitive) Ratgeber die Rolle einer testimonialen Autorität einnehmen.

\section{KOGNITIVE ÜBERLEGENHEIT UND DAS „AUTORITÄTEN- RESSOURCEN-MODELL“ PERSONALER WEISHEIT}

Jason Swartwood (2013) hat unlängst eine Theorie der personalen Weisheit vorgeschlagen, die ähnlich wie der vorliegende Ansatz den Aspekt der Entscheidungsfindung in den Mittelpunkt rückt und zudem eine klare Vorstellung entwickelt, von welcher Art die kognitive Leistung ist, die weise Personen erbringen müssen. Gemäß dem von ihm vertretenen „Expert Skill Modell of Wisdom" (ESMW) beinhaltet personale Weisheit eine kognitive Leistung, die darin besteht, dass man versteht, wie man sich in einem für Weisheit relevanten Bereich verhält (understanding how to conduct oneself). ${ }^{25}$ Swartwood sagt wenig über die Form dieses Verstehens, d.h. von welcher Art die kognitive Einstellung des Verstehens ist. Er geht jedoch davon aus, dass die kognitive Leistung, die mit einem solchen für Weisheit relevanten Verstehen verbunden ist, vom dem Typ her genau dieselbe ist, die der Entscheidungsfindung von Experten zugrunde liegt. Seiner These zufolge besitzen Experten die Fertigkeit, im Bereich der Expertise die richtigen Entscheidungen treffen zu können (expert decision-making skill). Diese Fertigkeit beruht auf einer Menge von individuellen Eigenschaften, ${ }^{26}$ die identisch sein soll mit derjenigen Menge

\footnotetext{
${ }^{25}$ Jason Swartwood geht davon aus, dass ein Bereich nur dann im Hinblick auf Weisheit relevant ist, wenn dieser zwei Merkmale aufweist: Der Bereich muss komplexe Wahlmöglichkeiten (complex choice) beinhalten und die in diesem Bereich zu treffenden Entscheidungen müssen so sein, dass sie den Akteur vor eine kognitive Herausforderung stellen, d. h. die Entscheidung muss mit einer nachhaltigen Koordination seines kognitiven Gesamthaushalts - sowohl im Hinblick auf konative als auch auf affektive Einstellungen - verbunden sein. Vgl. Jason D. Swartwood, „Wisdom as an Expert Skill," Ethical Theory and Moral Practice 16, Nr. 3 (2013): 513 f.

${ }^{26}$ Nach Swartwood, „Wisdom,” 519 hat die empirische Forschung zur Entscheidungskompetenz von Experten gezeigt, dass Personen die sich in Bereichen mit komplexen Wahlmöglichkeiten durch anspruchsvolle Leistungen hervortun, über ein spezielles Set von Fähigkeiten verfügen müssen: (i) Intuitive Fähigkeiten: Experten können oft mühelos ohne bewusste Überlegung erkennen, was sie tun sollen. (ii) Deliberative Fähigkeiten: Experten können durch sorgfältige und
} 
von Eigenschaften, auf die sich weise Personen stützen können, wenn sie entsprechende Entscheidungen treffen.

Wenn diese These zutreffend ist, beruht die kognitive Überlegenheit, die weise Personen als Ratgeber auszeichnet, nicht wie im vorliegenden Ansatz auf der besonderen epistemischen Position, die ein weiser (kognitiver) Ratgeber als testimoniale Autorität im interpersonalen Zusammenhang einer Sprecher-HörerInteraktion einnimmt. Stattdessen wird angenommen, dass kognitive Überlegenheit aufgrund der besonderen Qualität des individuellen kognitiven Zustands zustande kommt, in dem sich eine weise Person befindet, wenn sie aufgrund von Expertenfähigkeiten die richtigen Entscheidungen trifft. Anders formuliert: Es sind nicht die kognitiven Ressourcen von testimonialen Autoritäten, sondern die individuellen Fertigkeiten von Experten, die weise Personen als kognitiv überlegene Personen auszeichnen.

Ich stimme Swartwood zu, dass Verstehen eine Schlüsselstellung einnimmt, wenn es um die Charakterisierung der Form von epistemischer Rationalität geht, die man von weisen Personen fordern muss. Ebenso ist es aus meiner Sicht völlig zutreffend, dass die Möglichkeit, richtige Entscheidungen treffen zu können, zur Grundlage der Angemessenheit der damit verbundenen kognitiven Einstellungen gehört. Dennoch vertrete ich in Bezug auf die Erklärung der kognitiven Leistung, die weise Personen zu erbringen haben - zumindest dann, wenn man in Betracht zieht, dass weise Personen in der Lage sein sollten, entsprechende Ratschläge erteilen zu können - eine gänzlich andere Auffassung. Der hier vorgeschlagene Ansatz geht nicht davon aus, dass die Art von kognitiver Leistung, die zur Zuschreibung personaler Weisheit berechtigt, mit den kognitiven Anstrengungen von Experten vergleichbar oder gar identisch ist.

Um zu sehen, warum Swartwoods These nicht greift, ist es wichtig, die folgende Unterscheidung zu berücksichtigen: Der Begriff der Autorität kann im Sinne von „being an authority" oder "having authority “ verwendet werden. ${ }^{27}$ Gemäß der ersten Verwendung liegt es nahe, die individuellen (epistemischen und praktischen) Eigenschaften von Personen zu betrachten, aufgrund der man ihnen den „Seins-Status“ einer kognitiv überlegenen Person zuschreiben kann. Orientiert man sich vorrangig an dieser ersten Begriffsverwendung (being an

langwierige Prozesse des Abwägens von Gründen herausfinden und bewerten, was sie tun sollen, wenn eine intuitive Identifikation fehlt oder nicht ausreicht. (iii) Metakognitive Fähigkeiten: Experten erkennen, wann und wie sie sich auf Intuition und/oder auf deliberative Prozesse verlassen können. (iv) Fähigkeit der Selbstregulierung: Experten erkennen, wie sie ihre Umgebung, ihr Verhalten und/oder ihre Motivation beeinflussen können, damit sie erfolgreich das tun können, was sie als notwendig erachten. (v) Fähigkeit zur Selbstkultivierung: Experten erkennen, wie sie ihre Praxis und ihre Erfahrungen anpassen müssen, um ihre intuitiven, deliberativen bzw. selbstregulierenden Fähigkeiten langfristig noch zuverlässiger machen zu können.

${ }^{27}$ Vgl. Joseph Raz, The Authority of Law: Essays on Law and Morality (Oxford University Press: 2009), 134. 
authority), ist die Versuchung groß, die kognitive Überlegenheit von Autoritäten in Analogie zur kognitiven Überlegenheit von Experten zu analysieren, nämlich ausschließlich in Bezug auf die individuellen Eigenschaften derjenigen Person, die im Sinne des Experten-Status eine bestimmte kognitive Leistung vollbringt. Diese Sichtweise liegt offenkundig Swartwoods Experten-Fertigkeiten-Modell personaler Weisheit zugrunde.

Es gibt jedoch Grund zur Annahme, dass diese erste Verwendungsweise unzureichend ist, wenn es darum geht, die besondere funktionale Rolle zu erfassen, die testimoniale Autoritäten im Zusammenhang mit kognitiven Ratschlägen spielen. Sobald wir fragen, inwiefern es möglich ist, dass eine Autorität epistemischen Einfluss auf eine kognitiv unterlegene Person nehmen kann (z. B. einen Laien), wird sichtbar, warum Vorschläge zur Analyse testimonialer Autoritäten, die den normativen Aspekt des „Habens“ von Autorität (having authority) ausklammern, ins Hintertreffen geraten. Um epistemischen Einfluss nehmen zu können, muss zwischen der kognitiv überlegenen Person (Autorität) und der kognitiv unterlegenen Person (Laie) eine unmittelbare Beziehung bestehen. Das gemeinsame Teilen einer solchen unmittelbaren Beziehung ${ }^{28}$ stellt im Rahmen der zweiten Begriffsverwendung eine notwendige Voraussetzung dafür dar, dass beide Seiten die wechselseitige epistemische Abhängigkeit ihrer kognitiven Situation gewahr werden. Ohne eine solche unmittelbare Beziehung ist die überlegene Person nicht in der Lage, die epistemischen Bedürfnisse der unterlegenen Person zu erfassen. Ohne ein solches Erfassen der epistemischen Bedürfnisse wird jedoch die unterlegene Person die ratgebende überlegene Person nicht als eine geeignete Quelle ihrer Erkenntnisbemühungen anerkennen. Letzteres ist jedoch eine unabdingbare Voraussetzung dafür, dass die überlegene Person (Ratgeber) mit ihrer kognitiven Aktivität normativen Einfluss nehmen kann.

Im Gegensatz dazu hängt der epistemische Status von Experten nicht vom Teilen einer solchen unmittelbaren Beziehung mit einer anderen Person ab. Auch Experten stehen natürlich in einer Relation zu anderen Personen, aber nur in einem sehr abstrakten Sinne, nämlich derart, dass sie mehr wahre und weniger falsche Überzeugungen oder ein tieferes Verstehen als die meisten anderen Personen in einem bestimmten Bereich besitzen. ${ }^{29}$ Für den Experten stellt es keine

\footnotetext{
${ }^{28}$ Die Tatsache, dass epistemische Autoritäten nur dann in der Lage sind, eine normative Kraft auszuüben, wenn sie in einer unmittelbaren Beziehung zum Laien stehen, hat Linda Zagzebski zum Anlass genommen, im Fall von Experten von einem ,very weak sense“ epistemischer Autorität zu sprechen: „The expert is an authority only in a very weak sense, since the expert and the layperson who defer to her may have no relationship with each other” (Zagzebski, „Epistemic,” 5). Das scheint mir aber immer noch, wie weiter unten gleich deutlich wird, eine zu starke Behauptung zu sein.

${ }^{29}$ Der Begriff des „Habens“ von Autorität ist in einer Weise relational, die für den Begriff des Experten offenkundig nicht gilt. Eine Person S kann in einem Bereich D relativ zu einer Person A eine Autorität sein und relativ zu einer anderen Person B nicht. Beispielsweise ist Jürgen Klopp
} 
notwendige Bedingung dar, die epistemischen Bedürfnisse anderer Person erfassen zu müssen. Man kann ganz offenkundig ein anerkannter Experte sein, ohne dass man sensitiv für die epistemischen Bedürfnisse irgendeines Laien ist. Mehr noch, im Vergleich zu epistemischen Autoritäten besteht für Experten das Gebot der Unabhängigkeit. Ein Experte, der sich in der Bewertung von Informationen ausschließlich an den epistemischen Bedürfnissen einer konkreten Person oder Gruppe von Personen orientieren würde, läuft automatisch Gefahr, seine epistemische Sorgfaltspflicht zu verletzen. Die Unabhängigkeit von den epistemischen Bedürfnissen einer bestimmten Person wird allgemein als Voraussetzung für die Objektivität des Expertenurteils angesehen. ${ }^{30}$

Vor diesem Hintergrund plädiere ich für einen alternativen Erklärungsansatz, der sich am Begriff des „Habens“ von Autorität (having authorithy) orientiert. Gemäß der vorgeschlagenen interpersonalen Erklärung testimonialer Rechtfertigung beruht der Entscheidungsprozess weiser Personen - sofern sie im Rahmen eines testimonialen Akts entsprechende Ratschläge artikulieren - auf kognitiven Ressourcen die über individuelle Expertenfertigkeiten hinausgehen. Sie sind vom selben Typ wie diejenigen kognitiven Ressourcen, die der Entscheidungs-

in Sachen Taktikwissen für die Spieler seiner Mannschaft eine Autorität, für einen anderen Trainer wie José Mourinho hingegen nicht. Beide Trainer sind jedoch Experten im Bereich der Fußballtaktik unabhängig davon, ob sie als Autoritäten akzeptiert werden. Autoritäten unterscheiden sich von Experten darin, dass sie in einer direkten Relation zu anderen Personen stehen. Um als testimoniale Autorität anerkannt zu werden, muss eine Person relativ zu anderen Personen in einer besseren epistemischen Position sein. Infolgedessen ist es möglich, dass eine Person $\mathrm{S}$ in ein und demselben Bereich relativ zu A eine testimoniale Autorität ist und relativ zu B keine testimoniale Autorität ist, nämlich dann, wenn $\mathrm{S}$ gegenüber $\mathrm{A}$, aber nicht gegenüber $\mathrm{B}$ in einer besseren epistemischen Position ist. Für Experten gilt das nicht. Wenn S als Experte in einem Bereich D allgemein anerkannt ist, ist es nicht möglich, dass S für A ein Experte ist und für B keine Experte ist. Natürlich kann es sein, dass man Experten, obwohl sie als solche anerkannt sind, trotzdem nicht vertraut. Der Begriff des Experten ist graduell. Experten können mehr oder weniger gut sein. Ob jedoch jemand ein Experte ist, hängt allein von der objektiven Charakterisierung seiner epistemischen Position innerhalb des betreffenden Bereichs ab. Dazu muss die epistemische Position des Experten ein objektives Mindestmaß an epistemischen Anforderungen erfüllen (z. B. muss die betreffende Person mehr wahre Überzeugungen besitzen als die meisten anderen Personen in diesem Bereich). Dieses objektive Mindestmaß liefert einen nicht-relationalen Grenzwert, ab wann jemand als Experte zählt.

${ }^{30}$ Darüber hinaus besteht zwischen Experten und epistemischen Autoritäten der folgende Unterschied: Die (testimoniale) Rolle des Experten bemisst sich für andere Personen daran, ob dieser eine verlässliche Informationsquelle ist. Hier scheint es sinnvoll zu sein, die Frage nach den kognitiven Ressourcen, auf die sich eine solche (verlässliche) Informationsquelle stützt, mit Blick auf die besondere Art der individuellen Fertigkeiten der betreffenden Person zu beantworten. Wir hatten jedoch bereits gesehen, dass sich der testimoniale Status einer epistemischen Autorität nicht darin erschöpft, eine verlässliche Informationsquelle zu sein. Epistemische Autoritäten sind in ihrer testimonialen Funktion darauf angewiesen von anderen Personen als vertrauenswürdig akzeptiert zu werden. Wenn diese Annahme zutreffend ist, sollte klar sein, dass im Fall von testimonialen Autoritäten andere kognitive Ressourcen im Spiel sind. 
findung einer bestimmten Klasse von testimonialen Autoritäten zugrunde liegt, nämlich solchen Autoritäten, die in der Lage sind beim Adressaten ihrer testimonialen Aktivität ein signifikanteres Verstehen von Fragestellungen und Problemlösungen in dem für Weisheit relevanten Bereich zu fördern oder hervorzurufen. ${ }^{31}$ Ich nenne diesen Ansatz das „Autoritäten-Ressourcen-Modell“ der Erklärung personaler Weisheit. Diesem Vorschlag liegt die allgemeine Idee zugrunde, dass eine Person, um ,weise“ genannt zu werden, von anderen Personen anerkannt werden muss. Das ist immer dann der Fall, wenn sie im Sinne einer testimonialen Autorität (zumindest potentiell) in der Lage ist, auf andere Personen normativen Einfluss nehmen zu können, indem sie die Funktion eines weisen (kognitiven) Ratgebers ausübt. Gemäß dieser Idee beruht die Entscheidungsfindung weiser ratgebender Personen auf kognitiven Ressourcen, die im Sinne der angeführten „Buck-Passing“ Erklärung testimonialer Rechtfertigung sozial verteilt sind, da ihnen ein arbeitsteiliger Prozess der gemeinsamen Kooperation von ratgebender Person (der testimonialen Autorität) und ratsuchender Person (dem Adressaten der testimonialen Aktivität) zugrunde liegt. Infolge dieses arbeitsteiligen Prozesses testimonialer Rechtfertigung hängt die Qualität der Entscheidungen, die im Zusammenhang mit weisen (kognitiven) Ratschlägen getroffen werden, nicht nur von individuellen Fähigkeiten der ratgebenden Person, sondern auch von den epistemischen Bedürfnissen und individuellen Fähigkeiten des Adressaten der testimonialen Aktivität ab, also derjenigen Person, an die der Ratschlag gerichtet ist.

Vor diesem Hintergrund ist die Frage nach den kognitiven Ressourcen für personale Weisheit neu zu bewerten. Das „Autoritäten-Ressourcen-Modell“ besagt, dass die Entscheidungsfindung von weisen (kognitiver) Ratgebern auf zwei unterschiedliche Arten von kognitiven Ressourcen beruht. Einerseits benötigen weise (kognitiver) Ratgeber spezielle adressaten-orientierte Fähigkeiten, um die epistemischen Bedürfnisse des Adressaten der testimonialen Aktivität erfassen zu können. Andererseits bedarf es neben den speziellen individuellen Fähigkeiten der ratgebenden Person auch so etwas wie kollektive kognitive Ressourcen. Denn aufgrund des arbeitsteiligen Prozesses der Rechtfertigung testimonialer Aktivitäten hängt der epistemische Erfolg des kognitiven Ratschlags wesentlich von der Art der Zusammenarbeit zwischen Sprecher und Höher ab. Entsprechend bedarf es allgemeiner Prinzipien und Mechanismen sozialer Kooperation, die die soziale Verteilung der epistemischen Verantwortung regeln

\footnotetext{
${ }^{31}$ Der hier verwendete Begriff der testimonialen Autorität steht im Einklang mit der Raz'schen „Service-Konzeption“ von praktischen Autoritäten, wonach ein Person A nur dann legitimiert ist, einer Person B etwas aufzuerlegen - z. B. eine Direktive zu befolgen -, wenn ihr Tun im Dienste von B ist, d. h. wenn das Befolgen der Direktive B hilft, Gründe für das eigene Tun zu erlangen, die besser sind, als wenn B selbst die Gründe hätte abwägen müssen. Die Rolle von Autoritäten ist nach dieser Konzeption nicht auf die bloße Übermittlung von Informationen beschränkt. Vgl. hierzu: Raz, The Morality of Freedom (Oxford: Clarendon Press, 1986); Raz, „The Authority“.
} 
und damit die Basis schaffen, dass die ratgebende Person (testimoniale Autorität) vom Adressaten der testimonialen Aktivität als geeignete Quelle seiner Erkenntnisbemühungen anerkannt wird.

Ausgehend von diesen Überlegungen soll nun abschließend im letzten Teil dieser Arbeit die Frage beantwortet werden, inwiefern Weisheit im Zusammenhang mit kognitiven Ratschlägen einen unverwechselbaren epistemischen Wert hat.

\section{DER WERT EPISTEMISCHER WEISHEIT}

Die grundlegende These dieser Arbeit lautet, dass Weisheit vom epistemischen Standpunkt aus betrachtet eine besondere testimoniale Aktivität ist - nämlich ein weiser kognitiver Ratschlag - die aufgrund ihrer interpersonalen Struktur einen unverwechselbaren epistemischen Wert besitzt. Die vorangegangene Diskussion gestattet es in dreierlei Hinsicht etwas mehr über diesen Wert zu sagen, nämlich (i) wie der Träger dieses Werts zu bestimmen ist, (ii) worin die Besonderheit der Wertschätzung und das damit verbundene Ziel der Untersuchung besteht, und (iii) welche axiologischen Konsequenzen es hat, dass weise (kognitive) Ratgeber testimoniale Autoritäten sind, deren epistemischer Erfolg von einer sozialen Verteilung der kognitiven Anstrengungen abhängt.

Gemäß den eingangs vorgenommenen Unterscheidungen muss zwischen dem individuellen (metakognitiven) Zustand des weisen (kognitiven) Ratgebers und seiner epistemischen Position getrennt werden. Der Träger des Werts der Weisheit ist nicht der individuelle (mentale) Zustand der ratgebenden Person, sondern die epistemische Position, in der sich eine Person befindet, wenn sie die interpersonale Rolle ausübt, dem Adressaten einer testimonialen Aktivität als „secondhand“ Quelle seiner Erkenntnisbemühungen zu dienen. Die epistemische Position des Ratgebers ist individuell betrachtet ein metakognitiver Zustand, der als integraler Bestandteil einer Sprecher-Höherer-Interaktion in die interpersonale Rolle einfließt und nur vermittelt über diese interpersonale Rolle eine epistemische Signifikanz erlangt. Gemäß der von mir diskutierten (ontologischen) Besonderheiten ist die epistemische Position des weisen Ratgebers an normative Bedingungen der angemessenen Artikulation eines weisen Ratschlags geknüpft. Eine Person ist nur dann in der epistemischen Position, ein weiser (kognitiver) Ratgeber zu sein - im Sinne des Wertträgers -, wenn sie in der Lage ist, die Gründe zu erfassen, die ihren Ratschlag rechtfertigen und sich der normativen Verpflichtungen bewusst ist, die mit den betreffenden Sprecher-HörerInteraktionen verbunden sind.

Die epistemische Position des weisen Ratgebers ist in zweierlei Hinsicht mit einer kognitiven Leistung verbunden: In der Perspektive der ratgebenden Person stellt der weise (kognitive) Ratschlag eine distinkte individuelle Leistung dar, da der Rategeber als testimoniale Autorität eine Entscheidung treffen muss, was 
langfristig gesehen für den Adressaten der testimonialen Aktivität - im Hinblick auf dessen epistemischen Bedürfnisse - das Beste ist. Nur solche Informationen, die diese Parameter erfüllen, verleihen dem kognitiven Ratschlag das Prädikat „weise sein“. Epistemische Weisheit lässt sich in dieser Hinsicht nicht mit anderen epistemischen Einstellungen wie Wissen oder Verstehen vergleichen, weil es sich hier um eine propositionale Einstellung handelt, die aus einer metakognitiven Perspektive erfolgt. Unverwechselbar ist Weisheit im Zusammenhang mit kognitiven Ratschlägen aber auch dadurch, dass es der einzige Fall ist, in dem der epistemische Erfolg einer kognitiven Einstellung davon abhängt, dass die betreffende Person in der Lage ist, $z u$ entscheiden, welche Informationen übermittelt werden und welche besser zurückzuhalten sind.

Nach der vorliegenden Konzeption bemisst sich der epistemische Wert des weisen (kognitiven) Ratschlags aber nicht nur an der besonderen individuellen (metakognitiven) Leistung des Ratgebers. Wichtiger noch ist die Tatsache, dass die individuelle Leistung nur dann zum Tragen kommt, wenn sie ein integraler Bestandteil der epistemischen Position des Ratgebers ist. Nur wenn der Adressat der testimonialen Aktivität bereit ist, die individuelle (metakognitive) Einstellung der ratgebenden Person als Quelle seiner Erkenntnisbemühungen anzuerkennen, erlangt die epistemische Position des Ratgebers einen epistemischen Wert. Auch der Adressat der testimonialen Aktivität muss daher etwas leisten - nämlich dem Sprecher der testimonialen Aktivität sein Vertrauen aussprechen, d.h. ihn als testimoniale Autorität akzeptieren -, damit die epistemische Position des weisen Ratgebers als Quelle der Erkenntnisbemühungen des Adressaten einen epistemischen Wert erlangt.

Die eben angesprochene Interdependenz von kognitiven Leistungen, die notwendig mit der epistemischen Position des kognitiven Ratgebers verbunden ist, sorgt dafür, dass die Aktivität des weisen (kognitiven) Ratschlags, sofern sie erfolgreich ist, einen unverwechselbaren epistemischen Wert hat. Selbst wenn wir annehmen würden, dass im Hinblick auf die individuelle kognitive Leistung kein Unterschied bestünde, ob wir es mit einem weisen (kognitiven) Ratgeber, einem Wissenden oder einem Verstehenden zu tun haben, sorgt die Interdependenz der kognitiven Leistungen dafür, dass die Analyse des epistemischen Werts der Weisheit nicht mit der von anderen epistemischen Werten vergleichbar ist. Es kommt an dieser Stelle ein interpersonaler Aspekt der Bewertung des epistemischen Erfolgs kognitiver Ratschläge ins Spiel, der weit über bisherige Analysen hinausgeht.

Zudem wurde im Rahmen des vorliegenden Ansatzes ein „AutoritätenRessourcen-Modell“ der Erklärung personaler Weisheit vorgeschlagen. Demnach ist die kognitive Leistung, die ein weiser (kognitiver) Ratgeber im Entscheidungsprozess erbringen muss, auf kognitive Ressourcen gestützt, die vom selben Typ sind wie diejenigen, die der Entscheidungsfindung einer ganz bestimmten Klasse von testimonialen Autoritäten zugrunde liegt, nämlich solchen 
Autoritäten, die beim Adressaten des Ratschlags ein signifikantes Verstehen in dem für Weisheit relevanten Bereich hervorbringen oder fördern können.

Mit diesem Vorschlag sind zwei weitreichende axiologische Konsequenzen verbunden: Zum einen geht der vorliegende Ansatz davon aus, dass es in Fällen des weisen (kognitiven) Ratschlags in erster Linie darum geht, dem Adressaten der testimonialen Aktivität ein signifikantes Verstehen der zugrundeliegenden Phänomene in dem für Weisheit relevanten Bereich zu vermitteln. Demnach ist die Wertschätzung der epistemischen Position des Ratgebers, sofern es um weise (kognitive) Ratschläge geht, nur dann angemessen, wenn die testimoniale Aktivität des Sprechers dem epistemischen Ziel des Verstehens dient, d. h. der Adressat der testimonialen Aktivität dazu gebracht wird, eine bestimmte (propositionale) Abhängigkeitsrelation (in der Art von ,p weil q“) - oder das Phänomen, das diese Relation repräsentiert - auf lange Sicht besser zu erfassen.

Zweitens besagt das „Autoritäten-Ressourcen-Modell“, dass epistemischer Erfolg im Fall des weisen (kognitiven) Ratschlags ein arbeitsteiliger Prozess ist, der auf einer gemeinsamen - durch soziale Kooperation hervorgebrachten - kognitiven Anstrengung von Sprecher und Hörer beruht. Gemäß dieser Annahme besitzt die individuelle (metakognitive) Einstellung des Ratgebers - genauso wie die individuelle (kognitive) Einstellung der Anerkennung des Ratgebers (als testimoniale Autorität) durch den Adressaten - im Erfolgsfall einen hinzukommenden kollektiven epistemischen Wert. Denn im Erfolgsfall hat nicht nur der Adressat einen epistemischen Gewinn im Bezug auf ein tieferes Verstehen des für Weisheit relevanten Bereichs zu verzeichnen. Umgekehrt profitiert auch der Ratgeber davon. Denn eine gelingende Kooperation festigt seine funktionale Rolle, im Hinblick auf den weisheitsrelevanten Bereich eine (anerkannte) testimoniale Autorität zu sein.

Angesichts der eben genannten Aspekte scheint die Behauptung, dass Weisheit im erkenntnistheoretischen Zusammenhang einen unverwechselbaren Wert hat, mehr als gerechtfertigt zu sein. Offen gelassen wird an dieser Stelle, wie weitreichend die Konsequenzen sind, die sich daraus für eine umfassende Analyse epistemischer Werte - die andere Arten von kognitiven Aktivitäten mit einschließt - ergeben. ${ }^{32}$

\footnotetext{
${ }^{32}$ Ich bedanke mich bei den Teilnehmern der Lodzener Weisheitstagung „Früherer und zeitgenössischer Sinn und Begriff von Weisheit" für die anregende Diskussion und produktive Atmosphäre der Tagung. Mein Dank gilt ebenso den Teilnehmern des Dresdner Forschungsseminars zum Thema „Weisheit“ für die vielen Stunden der kritischen Auseinandersetzung mit weiten Teilen dieses Textes und den damit verbundenen zahlreichen Anmerkungen. Der Deutschen Forschungsgemeinschaft ist für die finanzielle Gewährung einer Sachbeihilfe für das Projekt SCHO 631/7-1 „Eine mehrdimensionale Theorie der Weisheit" zu danken, in dessen Rahmen dieser Text entstanden ist.
} 


\section{BIBLIOGRAPHIE}

Ardelt, Monika. „Wisdom as Expert Knowledge System: A Critical Review of a Contemporary Operationalization of an Ancient Concept." Human Development 47, Nr. 5 (2004): 257-285.

Baier, Annette. „Trust and Antitrust”. Ethics 96, Nr. 2 (1984): 231-260.

Beahr, Jason. „Two Types of Wisdom”, Acta Analytica 27 (2012): 81-97.

- „Sophia: Theoretical Wisdom and Contemporary Epistemology." In Virtues and Their Vices, Hrsg. K. Timpe and C. Boyd, New York: Oxford University Press, 2014, 303-325.

Faulkner, Paul. „On Telling and Trusting.” Mind 116 (2007): 875-902. Knowledge on Trust. New York: Oxford University Press, 2011.

Fileva, Iskra und Tresan, Jon. „Wisdom Beyond Rationality: A Reply to Ryan.” Acta Analytica 28 (2013): 229-235.

Fricker, Elisabeth. „Telling and Trusting: Reductionism and Anti-Reductionism in the Epistemology of Testimony." Mind 104 (1995): 393-411.

Frost-Arnold, Karen. „The cognitive attitude of rational trust.” Synthese 191 (2014): 1957-1974.

Glück, Judith. Weisheit - Die 5 Prinzipien des gelingenden Lebens. München: Kösel-Verlag, 2016.

Grimm, Stephen. „Wisdom.” Australasian Journal of Philosophy 93 (2015): 139-154.

Hinchman, Edward. „Telling as Inviting to Trust.” Philosophy and Phenomenological Research 70 (2005): 562-587.

Holton, Richard. „Deciding to trust, coming to believe”. Australasian Journal of Philosophy 72, Nr. 1 (1994): 63-76.

Kyriacou, Christos. „Ought to Believe, Evidential Understanding and the Pursuit of Wisdom.” In Epistemic Reasons, Epistemic Norms, and Epistemic Goals, herausgegeben von Martin Grajner und Pedro Schmechtig, DeGruyter: Berlin \& Boston, 2016, 383-406.

McMyler, Benjamin. Testimony, Trust, \& Authority. Oxford University Press, 2011.

Miscevic, Nenad. „Wisdom, Understanding and Knowledge: A Virtue-Theoretic Proposal.” Acta Analytica 27 (2012): 127-144.

Moran, Richard. „Getting Told and Being Believed.” Philosophers' Imprint 5 (2005): 1-29.

Raz, Joseph. The Morality of Freedom. Oxford: Clarendon Press, 1986.

- The Authority of Law: Essays on Law and Morality. Oxford University Press, 2009.

Reid, Thomas. An Inquiry into the Human Mind, Penn State University Press, 1997.

Riggs, Wayne. „Understanding 'virtue' and the virtue of understanding.” In Intellectual virtue: perspectives from ethics and epistemology, herausgegeben von Michael DePaul and Linda Zagzebski, Oxford University Press, 2003, 203-226.

Roberts Robert C. und Wood W. Jay. Intellectual Virtues: An Essay in Regulative Epistemology. Oxford University Press, 2007.

Ryan, Shane. „Wisdom: Understanding and the Good Life.” Acta Analytica 31, Nr. 3 (2016): 235-251.

Ryan, Sharon. „What is Wisdom?”. Philosophical Studies 93 (1999): 119-139.

. „Wisdom, Knowledge, and Rationality.” Acta Analytica 27 (2012): 99-112.
. „A Deeper Defense of the Deep Rationality Theory of Wisdom: A Reply to Fileva and Tresan.” Acta Analytica 32, Nr. 1 (2017): 115-123.

Schmechtig, Pedro. „Ist Weisheit ein epistemisches Ziel?”. Zeitschrift für Philosophische Forschung 69, Nr. 4 (2015): 529-549.

_. „Epistemische Werte.” In Handbuch Erkenntnistheorie, herausgegeben von M. Grajner \& G. Melchior, Stuttgart: Metzler, im Druck 2019, 286-294.

Shogenji, Tomoji. „A Defense of Reductionism about Testimonial Justification of Beliefs.” Nous 40 (2006): 331-346.

Swartwood, Jason D. „Wisdom as an Expert Skill.” Ethical Theory and Moral Practice 16, Nr. 3 (2013): 511-528. 
Tiberius, Valerie. The Reflective Life - Living Wisely within our Limits. Oxford University Press, 2008.

Whitcomb, Denis. „Wisdom.” In Routledge companion to epistemology, herausgegeben von

S. Bernecker and D. Pritchard, London: Routledge, 2010, 95-105.

Zagzebski, Linda. Virtues of the mind. Cambridge University Press, 1986.

. Epistemic Authority - A Theory of Trust, Authority, and Autonomy in Belief. Oxford University Press, 2012.

\section{COGNITIVE ADVICE, TESTIMONIAL AUTHORITY AND THE VALUE OF EPISTEMIC WISDOM}

This paper deals with the question of which role wisdom plays in epistemology. Firstly, I briefly sketch the general framework of this inquiry. Linked to this is a specific proposal for the conceptual definition of epistemic wisdom. This proposal is explained in more detail with regard to three different aspects (ontological, epistemic, axiological) of the attribution of epistemic wisdom. In the following, the main thesis behind the proposed definition is discussed more closely. Accordingly, epistemic wisdom is a testimonial activity that has an interpersonal structure. As a consequence of this view, wise (cognitive) advisors are to be regarded as testimonial authorities whose cognitive superiority is to be analyzed within the framework of an "authority-resources model" of the explanation of personal wisdom. Finally, on the basis of this model, it can be shown that wisdom in connection with cognitive advice has a distinctive epistemic value.

\section{Keywords:}

cognitive advice, epistemic authority, epistemic values, epistemic wisdom, metaepistemology, personal wisdom, value of wisdom 